\title{
Hybrid RANS/LES Simulations for Aerodynamic and Aeroacoustic Analysis of a Multi-Element Airfoil
}

\author{
Bastian Nebenführ \\ Huadong Yao \\ Shia-Hui Peng \\ Lars Davidson
}

June, 2012

\begin{abstract}
A hybrid RANS/LES modeling approach is used for the simulation of the turbulent flow around a three-element airfoil in high-lift configuration. Based on the simulation outcome, a detailed analysis of the flow is performed. Moreover, a comprehensive aeroacoustic analysis involving all three elements of the airfoil is presented. In order to provide input data for acoustic analogies, the results of the simulation are sampled at a permeable stationary surface in the near-field of the airfoil as well as at the airfoil itself. With the help of both the Kirchhoff integral surface method, the Ffowcs-Williams and Hawkings method for a stationary, permeable surface and the Curle method, the far-field noise signature of the high-lift airfoil is computed. The Sound Pressure Level spectrum exhibits a broad banded shape with several narrow banded tonal peaks at low Strouhal numbers. Also the broad banded peak at high Strouhal numbers, which is typically associated with vortex shedding behind the blunt slat trailing edge was captured. With the help of Curle's acoustic analogy, the noise emission pattern of the three elements is explored isolated from each other, revealing that both slat and flap act as dipoles. By refining the used grid, the flow results are significantly improved in terms of slat shear layer instability and resolved turbulent content as compared to our previous work.
\end{abstract}

\section{Introduction}

During the take-off as well as during the approach and landing phases of a typical flight, so-called high-lift devices are deployed on the airplanes wings in order to compensate for the lower lift force at the reduced airspeed compared to cruising conditions. The high-lift devices also allow to operate efficiently (that is without stall) at higher Angles of Attack (AoA) than with a clean airfoil. A typical high-lift airfoil consists of a leading edge slat, a main airfoil and one or several trailing edge flaps. While the flaps create most of the additional lift, the slat is deployed in order to avoid flow separation on the suction side of the main wing element. If this flow separation occurs, it can lead to stall, which stands for a sudden loss of lift force and is hence a safety risk.

A consequence of deploying high-lift devices at airfoils is not only the increase of lift force, but also an increased generation of flow induced noise, which is referred to as airframe noise. This is an undesirable, but unavoidable, side-effect of today's high-lift devices. In fact, due to the introduction of new High-Bypass-Ratio engines, the engine noise could be reduced considerably. Consequently, the noise created by the high-lift system can match or even exceed the engine noise, particularly during approach and landing. As airports tend to be neighboring urban areas, noise emission from starting and landing aircraft has become a societal problem. In order to protect residents from the aircraft noise, more and more stringent noise emission regulations have been imposed on the aircraft industry [1]. The noise reduction of entire aircraft and in particular of the high-lift devices is hence a challenge of great industrial interest nowadays.

Research on the prediction and reduction of airframe noise stemming from the high-lift system has been carried out in many ways. Traditionally, wind tunnel experiments on isolated wings [2-4] or aircraft scale models [3-5] have been conducted. Also some fly-over noise measurements on an Airbus A340 were performed [6]. More recently, also numerical predictions using Computational Fluid Dynamics (CFD) were exercised [7-10]. Often only the near-field noise signature has been investigated numerically as in [11], but some authors also computed the far-field noise $[7,8]$.

It is nowadays widely acknowledged that particularly the slat dominates the overall generated noise $[2,3,11-$ 13] of a wing. Due to that, a lot of effort was focused on the investigation of slat noise and slat noise sources $[3,8,11,14-16]$. Two main noise generation mechanisms were identified at the slat, namely the noise generation due to the instabilities of the shear layer at the slat cusp and the vortex shedding behind the blunt trailing edge of the slat. Many research groups have in the past investigated the influence of the slat inclination angle 
$[7,14,17]$ and the state of the boundary layer on the slat [18]. Khorrami et al. [14] found in their numerical study that the vortex shedding behind the slat only occurred for a slat inclination of $30^{\circ}$. When testing a slat inclination of $20^{\circ}$, however, the vortex shedding disappeared. They attribute a loud tonal noise at frequencies of about $40 \mathrm{kHz}$ to the vortex shedding. Afterwards, Singer and co-workers [7] used the unsteady data from Khorrami's computations for far-field noise prediction based on the Ffowcs-Williams and Hawkings method. They could further evidence that indeed the far-field noise spectrum of the case with $30^{\circ}$ slat deflection featured a strong tonal noise at frequencies between 38 and $45 \mathrm{kHz}$ depending on the slat trailing edge thickness, whereas this tonal peak was not observed for the $20^{\circ}$ case.

Computational AeroAcoustics (CAA) are dependent on the availability of time-dependent and accurate information of the fluctuating flow field. Direct Numerical Simulation (DNS) and Large-Eddy Simulation (LES) would be ideal for providing the required input, but are out of reach for wall-bounded flows of industrial interest. At the same time, traditional Reynolds-Averaged Navier-Stokes (RANS) Simulations fail to provide the necessary unsteady information for aeroacoustic analysis. Common practice today is to use hybrid RANS/LES methods, which are able to combine the advantages of both LES and RANS, being able to accurately represent fluctuating flow properties on the one hand and efficient modeling of the boundary layers on the other hand. A multitude of approaches has emerged in the past, of which the most prominent is the so-called Detached-Eddy Simulation (DES) proposed by Spalart [19]. The idea of DES is to use RANS in the attached boundary layer, where it is well adjusted and evaluated and to use LES in separated flow regions. In the separated regions, the flow is dominated by large scale turbulence, which can efficiently be resolved using LES even on a coarse mesh.

Phenomena that always are present in flows around wings in high-lift configuration are, amongst others, laminar-turbulent boundary layer transition, free shear layers, wakes, thick boundary layers and the interaction of those. Furthermore, flow separation over the flap can occur already at moderate AoA. The presence of the above mentioned phenomena makes the flow around a high-lift configuration a very complex one. It is important to take into account and accurately represent all the flow characteristics, because failure in predicting one of the phenomena may compromise the prediction of another phenomenon. Consequently the integrity of the entire simulation is jeopardized. Hence, this complexity makes the flow around a high-lift airfoil a challenging test case for the evaluation of turbulence modeling. Recently, Deck [20,21] has demonstrated the feasibility of a hybrid RANS/LES modeling approach for the prediction of high-lift flows. In his simulations, he employs a zonal DES approach in which designated DES and RANS zones are specified by the user [22]. Also Choudhari and Khorrami [11] and Lockard and Choudhari [8] have used a hybrid RANS/LES approach. Essentially, they use unsteady RANS with Menter's SST $k-\omega$ model $[23,24]$ in their computations. In order to eliminate the highly dissipative effects of the turbulence model, the turbulence production term was switched off in the slat cove region. Thus, the strategy can be interpreted as being similar to a zonal implicit LES [25-27].

In the present work, the turbulent flow around a multi-element airfoil with deployed high-lift devices is investigated. The main objectives of this work are twofold. Firstly, the far-field noise signature of the airfoil is to be determined. For that purpose acoustic analogies are used, which are supported by the instantaneous and non-linear input from the hybrid RANS/LES simulation. The acoustic analogies used are, namely, the Kirchhoff integral method [28], the Ffowcs-Williams and Hawkings method [29,30] for a stationary, permeable surface and Curle's method [31]. A comparative study of the results obtained with the different acoustic analogies is provided. Unlike most research in literature, the present work specifically accounts for the noise generated by all three elements and not only by the slat. Using the acoustic analogy of Curle makes it possible to explore the noise emission behavior of the isolated elements. As a second objective, the performance of the used hybrid RANS/LES modeling approach in predicting the flow around the airfoil is assessed. The calculations performed in the present work utilize the algebraic hybrid RANS/LES model designed by Peng [32, 33].

\section{Test case description}

The DLR F15 three-element high-lift airfoil is studied as a typical example of an airfoil in high-lift configuration. The same geometry has previously been subject to investigation in the LEISA (Low noise Exposing Integrated design for Start and Approach) project [12,13] at DLR (German Aerospace Center). During the course of this project, an experimental campaign has been carried out in the atmospheric low-speed wind tunnel (DNW-NWB) in Braunschweig, Germany, which resulted in experimental surface pressure data. More recently, the same geometry has been a test case in the ATAAC (Advanced Turbulence Simulation for Aerodynamic Application Challenges) EU project and was calculated by numerous partners. 


\subsection{Hybrid RANS/LES computation}

The investigated airfoil consists of a main wing with a leading edge slat and a trailing edge flap. The deflection angles of the slat and the flap are $28.8^{\circ}$ and $38.3^{\circ}$, respectively, which gives rise to the anticipation of narrow banded tonal noise as found by for example Khorrami et al. [14]. All trailing edges of the three elements of the airfoil are blunt, except the slat cusp, which is artificially sharpened.

Numerical simulations of the DLR F15 airfoil have been carried out using a hybrid RANS/LES LES approach. The method applied uses an algebraic mixing-length model in the RANS region, which is combined with the Smagorinsky model [34] in the LES region. Due to its simple algebraic nature and the absence of a transport equation for modeled turbulent quantities, one can refer to the model as a zero-equation hybrid model. In the following, the model will be termed HYB0 [32, 33].

In contrast to the wind tunnel measurements, the airfoil is here simulated at free flight, i.e. without the influence of the wind tunnel side walls. The wind tunnel tests were carried out at an AoA of $\alpha=7.05^{\circ}$, which had to be corrected numerically in order to take into account the blockage stemming from the flow separation on the wind tunnel side walls. This correction was done in terms of altering the AoA in RANS simulations so as to match the experimental surface pressure distribution. A detailed study using different RANS models and investigating amongst others the effect of the AoA and transition specification is presented in [35]. According to the RANS simulations, a corrected AoA of $\alpha=6^{\circ}$ has been chosen for the hybrid RANS/LES simulations. Furthermore, the transition from laminar to turbulent boundary layers is controlled on all three elements. More specifically, the upper and lower surfaces of the slat are treated as laminar, which is in agreement with the findings of Storms et al. [18], who observed that above a slat inclination of $19^{\circ}$ the slat boundary layer remained laminar. However, transition locations are specified on the pressure and suction sides of the main wing as well as on the suction side of the flap. The freestream Mach number and chord-based Reynolds number are $M_{\infty}=0.15$ and $R e_{\infty}=2.1 \times 10^{6}$, respectively.

In a previous work [36], computations with the HYB0 model on the same geometry were performed and especially the importance of the grid resolution in the $x-y$ plane was pointed out. Hence, a refined grid was generated and is used in this present effort. The grid is of hybrid character using hexahedral cells near the walls in order to resolve the boundary layers and using tetrahedral cells away from the wall. The geometry under consideration is a two-dimensional wing section of a generic aircraft used in the LEISA project and hence, the grid was build in a 2D plane first. The two-dimensional slice consists of about 200000 grid points in the $x-y$ plane. In order to create three-dimensionality, 160 of those 2D grid slices are lined-up equidistantly in spanwise direction over a distance of $16 \%$ of the stowed chord-length, $C$. The resulting three-dimensional wing section is unswept and the grid consists of a total of $32 \times 10^{6}$ grid points with a spanwise resolution of $\Delta z / C=0.001$. In the far-field, the boundary of the domain is located at a distance of $100 C$ from the airfoil. In spanwise direction, periodic boundary conditions are employed.

In the simulations, a timestep of $\Delta t=5 \times 10^{-6} \mathrm{~s}$ has been employed in order to ensure a CFL number based on the maximum speed of an acoustic wave, $\left(U_{\infty}+a\right)$, of $C F L<4$ in the slat cove region.

Table 1: Information about computational cases

\begin{tabular}{ccccccc}
\hline \hline Case & Grid & $N\left[10^{6}\right]$ & $\Delta x_{\min } / C$ & $L_{z}$ & $\Delta z / C$ & Model \\
\hline Case1 & original (3D) & 32 & 0.0015 & $0.16 C$ & 0.001 & HYB0 model \\
Case2 & refined (3D) & 32 & 0.0005 & $0.16 C$ & 0.001 & HYB0 model \\
Case3 & original (2D) & 0.2 & 0.0015 & - & - & SST RANS \\
\hline
\end{tabular}

All results presented in Section 3 were obtained with the HYB0 model on the refined grid (Case2) as mentioned above. For comparison, some results from an older computation with the same hybrid RANS/LES model on the original, but coarser grid (Case1) will also be included. Along with the two turbulence resolving computations, also the velocity profiles of a 2D RANS with Menter's SST $k-\omega$ model $[23,24]$ are presented as a reference (Case3). Details about the different cases are given in Table 1.

To enable analysis of the far-field sound via the acoustical analogies of Kirchhoff and Ffowcs-Williams and Hawkings, a permeable integral surface is included into the grid. The surface follows the geometry quite closely and then stretches about $6 C$ downstream of the flap trailing edge in order to include the noise created by the separated flow behind the airfoil. The distance of the surface from the airfoil is chosen in a way that it is including all the acoustical sources stemming from the core flow region. This is ensured by investigating the vorticity magnitude of a precursor RANS calculation, according to the method proposed by Yao et al. [9]. In the downstream direction, the integral surface is kept open in order to reduce the artificial noise induced by vortices passing through the surface. 


\subsection{Acoustic Analogies}

In acoustics, one is usually interested in the noise that is radiated to a far-field observer. As the present case can be understood as the situation of a landing airplane, the observers would most likely be residents near an airport. The typical distance from the plane to the ground will vary during approach and landing, but will be in the order of a couple of hundreds of meters.

As today, it is popular to decouple the acoustic analysis from the flow computation. Initially, CFD is used for providing the near-field flow solution, which includes all the noise sources and captures the non-linear flow effects. In a second step, acoustic analogies are used for computing the noise radiation to the far-field observers. Three acoustic analogies have been employed in studying the noise signature of the present airfoil configuration, namely the Kirchhoff surface integral method [28], the Ffowcs-Williams and Hawkings method [29, 30] for a stationary permeable surface and the Curle method for a stationary solid surface [31]. A brief description of the employed analogies follows below.

\subsubsection{Kirchhoff surface integral method}

Kirchhoff's theory [37] was originally used for describing electromagnetic waves. Later on, Kirchhoff's formula has been exploited for acoustical problems on stationary and moving integral surfaces [28]. In Kirchhoff's method, all non-linear effects of the acoustical sources are assumed to be enclosed by a permeable integral surface. While all acoustic sources are enclosed by the surface, the flow field outsides the surface satisfies the linear condition so that the homogeneous wave equation is fulfilled. With the help of generalized derivatives $[28,30]$, the Kirchhoff equation for a stationary surface [38] reads as follows:

$$
\bar{\square}^{2} p^{\prime}(\vec{x}, t)=-\frac{\partial p^{\prime}}{\partial n} \delta(f)-\frac{\partial}{\partial x_{i}}\left[p^{\prime} n_{i} \delta(f)\right]
$$

In Eq. (1), generalized derivatives are denoted by an overbar, as for the wave operator in $\square^{2}, \delta(f)$ is the Dirac delta function, $f$ is the surface itself and $n_{i}$ denotes the components of the unit normal vector pointing outwards from the surface. A solution to Eq. (1) can be found via the Green function in free space.

$$
\begin{aligned}
p_{K}^{\prime}(\vec{x}, t)= & \frac{1}{4 \pi} \int_{f=0}\left(\frac{\cos \theta}{R^{2}}\left[p^{\prime}(\vec{y}, t)\right]_{\tau}-\frac{1}{R}\left[\frac{\partial p^{\prime}(\vec{y}, t)}{\partial n}\right]_{\tau}\right. \\
& \left.+\frac{\cos \theta}{c_{0} R}\left[\frac{\partial p^{\prime}(\vec{y}, t)}{\partial \tau}\right]_{\tau}\right) d S
\end{aligned}
$$

where $R$ denotes the distance to the observer, i.e. $R=|\vec{r}|=|\vec{x}-\vec{y}|, \cos \theta=\left(r_{i} / R\right) n_{i}, c_{0}$ is the speed of sound and []$_{\tau}$ indicates that the term is evaluated at retarded time $\tau=t-R / c_{0}$. In the present analysis, the three terms of the surface integral will be treated as three individual surface integrals [10].

$$
\begin{aligned}
& p_{1, K}^{\prime}(\vec{x}, t)=\frac{1}{4 \pi} \int_{f=0}\left(\frac{\cos \theta}{R^{2}}\left[p^{\prime}(\vec{y}, t)\right]_{\tau}\right) d S \\
& p_{2, K}^{\prime}(\vec{x}, t)=-\frac{1}{4 \pi} \int_{f=0}\left(\frac{1}{R}\left[\frac{\partial p^{\prime}(\vec{y}, t)}{\partial n}\right]_{\tau}\right) d S \\
& p_{3, K}^{\prime}(\vec{x}, t)=\frac{1}{4 \pi} \int_{f=0}\left(\frac{\cos \theta}{c_{0} R}\left[\frac{\partial p^{\prime}(\vec{y}, t)}{\partial \tau}\right]_{\tau}\right) d S
\end{aligned}
$$

The three terms represent the noise due to pressure fluctuations, the gradient and the time derivative of the pressure fluctuations, respectively.

\subsubsection{Ffowcs-Williams and Hawkings method (FWH) for a stationary, permeable surface}

The acoustic analogy developed by Ffowcs-Williams and Hawkings [29] was derived for solid surfaces moving at an arbitrary speed, $v_{n}$. Here, the special case of a stationary and permeable surface is used, which can easily be extracted from Brentner's formulation [30] by setting $v_{n}=0$.

Brentner and Farassat [30] rewrote the compressible Navier-Stokes equations with the help of generalized functions into a generalized wave equation with non-linear source terms, which is also called the FWH-Equation 
(Eq. (4)).

$$
\begin{aligned}
\bar{\square}^{2} p^{\prime}(\vec{x}, t)= & -\frac{\partial}{\partial x_{i}}\left[\left(p^{\prime} \delta_{i j}+\rho u_{i} u_{j}\right) n_{j} \delta(f)\right] \\
& +\frac{\partial}{\partial t}\left[\rho u_{j} n_{j} \delta(f)\right] \\
& +\frac{\bar{\partial}^{2}}{\partial x_{i} x_{j}}\left[T_{i j} H(f)\right]
\end{aligned}
$$

where $T_{i j}=\left(p^{\prime}-\rho^{\prime} c_{0}^{2}\right) \delta_{i j}-\tau_{i j}+\rho u_{i} u_{j}$ is the Lighthill tensor [39, 40], $\delta_{i j}$ is the Kronecker delta and $H(f)$ is the Heaviside function. As described for the Kirchhoff method, we assume the surface to enclose the entire core flow region. Due to that assumption, it is possible to neglect the volume integral including the Lighthill tensor for the FWH method. A solution to Eq. (4) can be found, once again, based on Green's function for free space.

$$
\begin{aligned}
p_{F}^{\prime}(\vec{x}, t)= & -\frac{1}{4 \pi} \frac{\partial}{\partial x_{i}} \int_{f=0}\left(\frac{1}{R}\left[p^{\prime} \delta_{i j}+\rho u_{i} u_{j}\right]_{\tau} n_{j}\right) d S \\
& +\frac{1}{4 \pi} \frac{\partial}{\partial t} \int_{f=0}\left(\frac{1}{R}\left[\rho u_{i} n_{j}\right]_{\tau}\right) d S
\end{aligned}
$$

The above solution to the FWH equation, is the one originally derived for solid surfaces neglecting the volume integral. In this formulation, the first and second integral can directly be understood in physical terms as the loading and the thickness noise, respectively $[30,41]$. When using a permeable surface, the terms lose their physical meaning and hence it is legitimate to write them as individual integrals as exercised by Yao et al. in [10]. In Eq. (6), it is then possible to interpret the three terms as the perturbations of pressure, momentum and mass through the surface, respectively.

$$
\begin{aligned}
& p_{1, F}^{\prime}(\vec{x}, t)=\frac{1}{4 \pi} \int_{f=0}\left(\frac{1}{R} \cos \theta\left[\left\{\frac{1}{c_{0}} \frac{\partial}{\partial \tau}+\frac{1}{R}\right\} p^{\prime}\right]_{\tau}\right) d S \\
& p_{2, F}^{\prime}(\vec{x}, t)=\frac{1}{4 \pi} \int_{f=0}\left(\frac{1}{R} \lambda_{i j}\left[\left\{\frac{1}{c_{0}} \frac{\partial}{\partial \tau}+\frac{1}{R}\right\}\left(\rho u_{i} u_{j}\right)\right]_{\tau}\right) d S \\
& p_{3, F}^{\prime}(\vec{x}, t)=\frac{1}{4 \pi} \int_{f=0}\left(\frac{1}{R} n_{j}\left[\frac{\partial}{\partial \tau}\left(\rho u_{j}\right)\right]_{\tau}\right) d S
\end{aligned}
$$

where $\lambda_{i j}=\left(r_{i} / R\right) n_{j}$.

\subsubsection{Curle's method for a stationary solid surface}

Curle's method can be regarded as a generalization of Lighthill's analogy $[39,40]$ in the presence of stationary solid walls. Introducing generalized variables into the compressible Navier-Stokes equations and rewriting them in terms of a wave equation with non-linear source terms, leads to the following:

$$
\begin{aligned}
\bar{\square}^{2} p^{\prime}(\vec{x}, t)= & -\frac{\partial}{\partial x_{i}}\left[p^{\prime} n_{i} \delta(f)\right] \\
& +\frac{\bar{\partial}^{2}}{\partial x_{i} x_{j}}\left[T_{i j} H(f)\right]
\end{aligned}
$$

The solution to the Curle equation is given by the free space Green function as

$$
\begin{aligned}
p_{C}^{\prime}(\vec{x}, t)= & -\frac{1}{4 \pi} \frac{\partial}{\partial x_{i}} \int_{f=0}\left(\frac{1}{R}\left[p^{\prime}\right]_{\tau} n_{i}\right) d S \\
& +\frac{1}{4 \pi} \frac{\bar{\partial}^{2}}{\partial x_{i} x_{j}} \int_{V}\left(\frac{1}{R}\left[T_{i j}\right]_{\tau}\right) d V
\end{aligned}
$$

In Eq. (8), the surface integral stands for the pressure fluctuations on the solid wall due to the turbulent boundary layer. The second term represents the impact of the fluctuating stresses on the sound field. Due to the numerically difficult evaluation of the volume integral term of Eq. (8), only the surface integral term 
is included here. We obtain the total noise for the Curle analogy then in the form presented by Yao and co-workers $[9,10]$ :

$$
p_{C}^{\prime}(\vec{x}, t)=\frac{1}{4 \pi} \int_{f=0}\left(\frac{1}{R} \cos \theta\left[\left\{\frac{1}{c_{0}} \frac{\partial}{\partial \tau}+\frac{1}{R}\right\} p^{\prime}\right]_{\tau}\right) d S
$$

It is noteworthy that Eq. (9) is the same as Eq. (6a) in the present formulation of the FWH approach. Hence, the FWH approach falls back to Curle's method, if a solid, stationary surface is regarded.

\section{Results and discussion}

In this section, firstly, aerodynamic results are presented, which are followed by an aeroacoustic analysis.

\subsection{Aerodynamic analysis}

\subsubsection{Instantaneous flow}

Initially, computations were performed with the HYB0 model on the original grid, which showed that the shear layer emanating from the slat cusp was showing strongly delayed instabilities [36]. The first Kelvin-Helmholtz instabilities could be observed only at about half the way to the impingement point on the lower wall of the slat trailing edge. This phenomenon was partly blamed on insufficient grid resolution in the $x-y$ plane and partly on the so-called "gray area" problem in hybrid RANS/LES methods. At the interface between RANS and LES, a gray area can be found in which the computation cannot be considered RANS nor LES. The gray area issue is for instance described by Spalart in [42]. In order to highlight the grid influence, a new grid with a refined slat cove region has been used here. Figure 1 depicts isosurfaces of the Q-invariant for the slat shear layer. Almost immediately after the slat cusp, the development of Kelvin-Helmholtz instabilities sets in, represented by the roll-up of coherent vortical structures in spanwise direction. However, the coherent structures are transformed into fully three-dimensional turbulence rather late. This delay in secondary instabilities can be attributed to the gray area and has to be addressed in terms of hybrid RANS/LES modeling rather than in terms of grid refinement. Generally, the grid refinement led to much richer resolved turbulence structures inside the recirculation zone of the slat cove. It can be seen that the structures are to some extent incorporated in the recirculating flow in the slat cove, whereas the remainder of the structures is ejected from that zone through the gap between the slat and the main wing. Due to a significant flow acceleration in this region, the structures are being stretched into long streamwise tubes. Also vortex shedding from the blunt slat trailing edge can be identified in Fig. 1. In Fig. 2, the z-component of the instantaneous vorticity is shown at a mid-span cutplane.

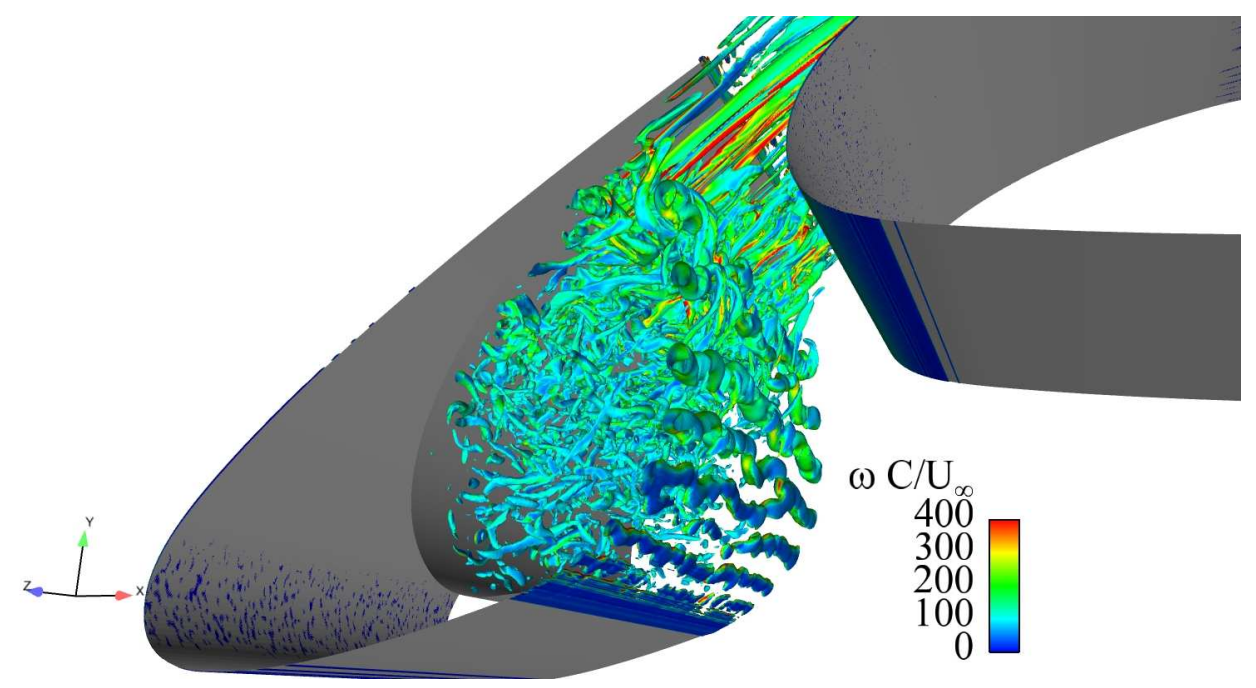

Figure 1: Isosurfaces of the Q-invariant for the free shear layer behind the slat cusp. $\frac{Q C^{2}}{U_{\infty}^{2}}=5000$. Coloring by vorticity magnitude.

A considerable delay in the formation of instabilities can be observed, which again should be addressed in terms of modeling. 


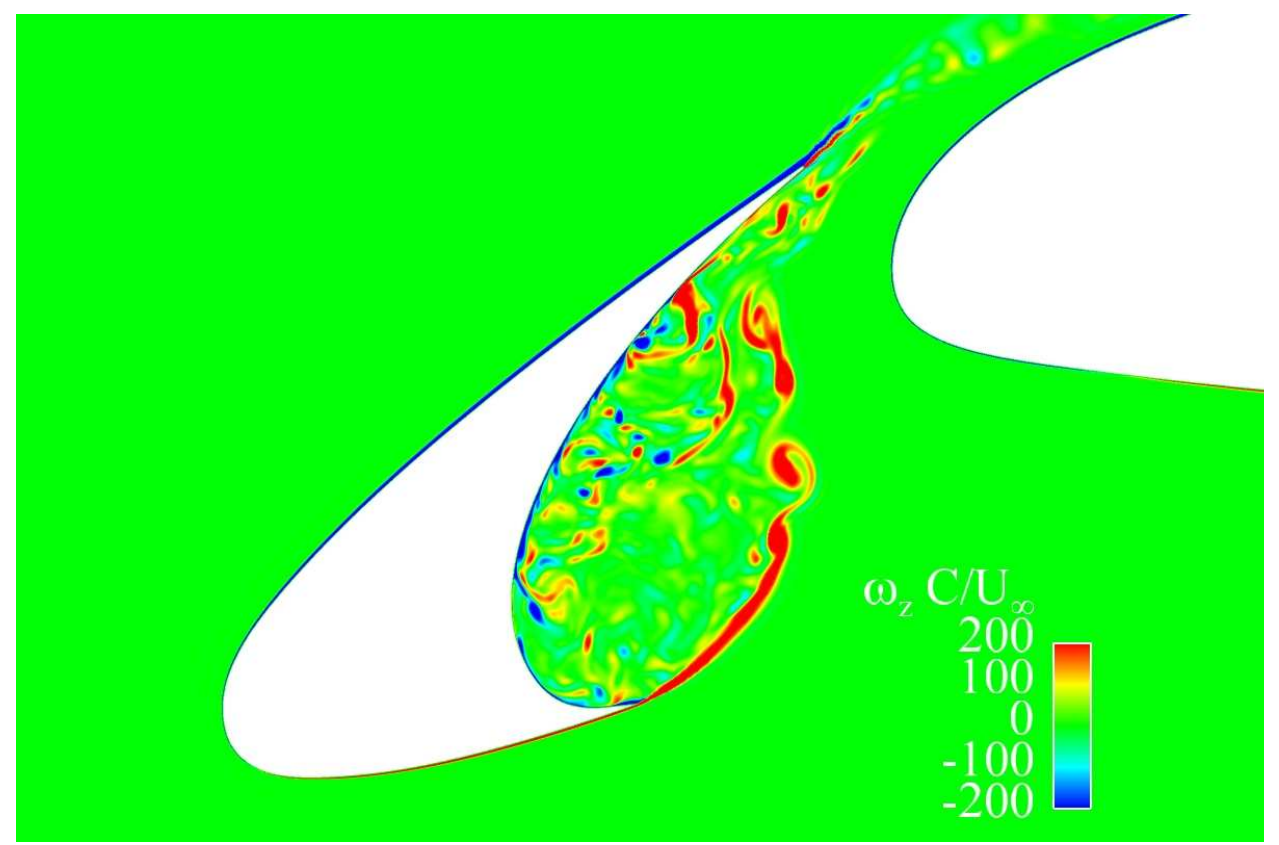

Figure 2: $z$-component of the normalized, instantaneous vorticity, $\frac{\omega_{z} C}{U_{\infty}}$, in the slat cove

\subsubsection{Mean flow}

The flow, as presented in Fig. 3, exhibits the topology of a typical flow around a high-lift airfoil. There are two recirculating regions in the slat cove and in the cove of the main wing. Besides that, flow separation occurs at this AoA on the flap as can be observed in Fig. 3(b).

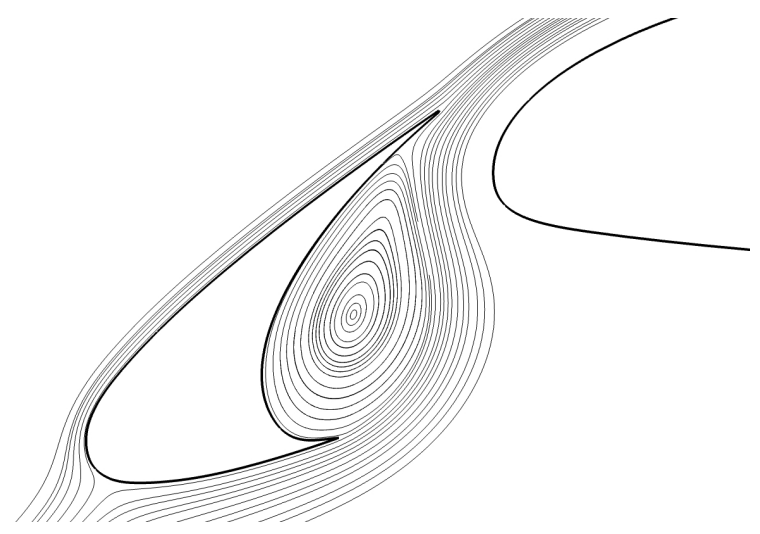

(a) slat

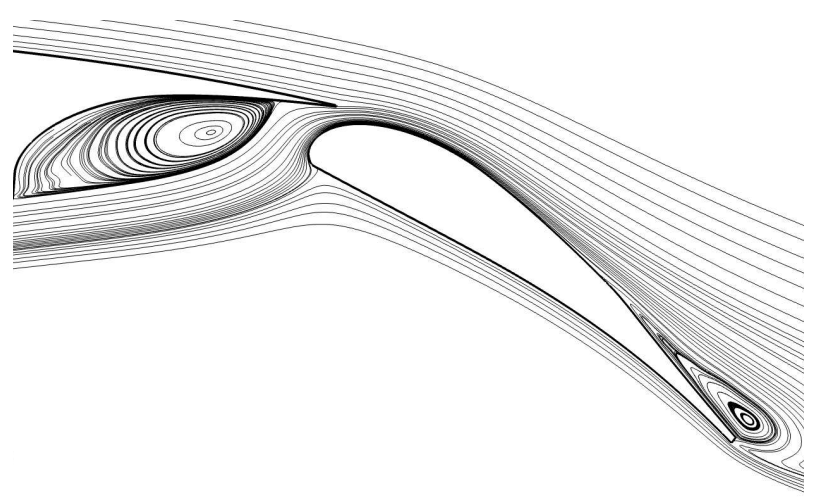

(b) flap

Figure 3: Flow pattern around the three-element airfoil, visualized by streamlines.

Experimental data is available for validation in form of the pressure distribution around the airfoils elements. The following plots contain a comparison between these experimental data and the simulation results. Please note that due to confidentiality the absolute pressure levels on the $y$-axis had to be omitted. Included in Figs. 4 and 5 are the results from both Case1 and Case2. In Fig. 4 all three elements are included and it can be seen that generally good agreement with the experiments is achieved on all elements in both cases. The only exception is the suction peak on the main wing, for which the pressure is somewhat underpredicted on both grids. Figure 5 provides a closer look at the pressure distributions around the slat and the flap. It reveals that the predictions of the pressure distributions around the slat are identical. The separated region on the suction side of the flap is represented better in Case2, i.e. Case1 suggests a plateau towards the trailing edge of the flap.

The streamwise component of the mean skin friction coefficient, $C_{f, x}$, is depicted in Fig. 6 . Case1 and Case2 produce similar distributions around all the elements, except for the suction side of the main wing, 
where Case 2 predicts consistently higher values. This is attributable to a higher turbulent content in the boundary layer for Case2, which might stem from the interaction of the slat wake with the boundary layer. Figure $6(\mathrm{~b})$ indicates that separation on the flap occurs at $x / C=1.028$ for Case1, whereas it occurs slightly downstream at $x / C=1.033$ for Case 2 .

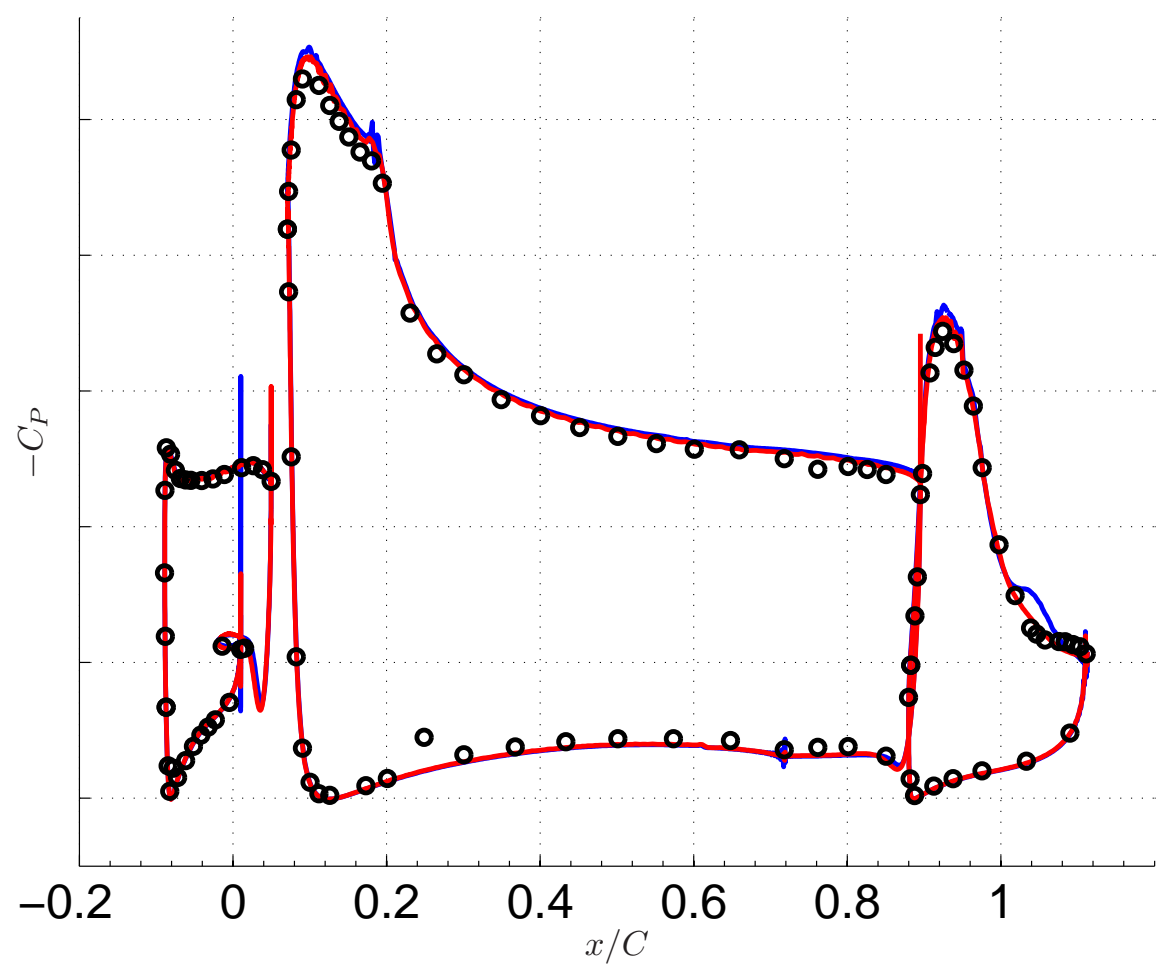

Figure 4: Mean pressure distribution for all elements. ——: Case1, — : Case2. ॰: Experiments. $\Delta C_{P}=1$

Time-averaged flow quantities have been extracted and compared at several locations around the slat and flap as presented in Figure 7. As mentioned before, previous simulations had shown strongly delayed instabilities of that free shear layer detaching from the slat cusp [36]. Hence, particular attention was payed to the treatment and development of that shear layer. Figure 8 displays a comparison of modeled and resolved shear stresses, $\overline{u_{n}^{\prime} u_{s}^{\prime}}$, for Case1 and Case2. It can be seen that at all four stations along the slat shear layer (locations given in Fig. 7(a)), the amount of resolved stresses exceeds the modeled ones. Moreover, comparing the HYB0 simulations on the two grids, one can see that Case2 enables greater shear stress levels to be resolved. Due to the fact that higher stress levels are resolved, also greater levels of turbulent kinetic energy in the slat shear layer are resolved with the grid of Case2. This is evidenced by Fig. 9(a), which shows the development of resolved turbulent kinetic energy along the shear layer from the slat cusp to the impingement point at the lower surface of the slat trailing edge. The curve exhibits that $k_{u v}$ is increasing linearly with increasing distance from the slat cusp. Only near the impingement point $\left(s / s_{\max }=1\right)$, a further, non-linear increase is shown. This can be attributed to the complex flow situation around the impingement location, as highlighted by Fig. 1. Note that the displayed kinetic energy is based on the normal stresses in the $x$ - and $y$-direction only, i.e. $k_{u v}=\sqrt{\overline{u^{\prime} u^{\prime}}+\overline{v^{\prime} v^{\prime}}}$. This has been done in order to be better able to compare with computational and experimental results in the literature. Lockard and Choudhari [8] present a numerical investigation of a similar multi-element airfoil. They show in a plot similar to Fig. 9(a) that the turbulent kinetic energy based on the normal stresses in all three directions, i.e. $k_{u v w}$, does not increase linearly from the slat cusp. In the present case, however, the normal stress in spanwise direction is so weak in the initial shear layer that there is virtually no difference between the curves of $k_{u v}$ and $k_{u v w}$. Deck [21] reports levels of resolved turbulent kinetic energy in the slat shear layer obtained with the Zonal DES (ZDES) [22] approach of about $2 \%$ for the same geometry. Also Lockard and Choudhari [8] show levels of $k_{u v} / U_{\infty}^{2} \approx 0.02$ in the shear layer for simulations of a similar geometry. The observations of Deck and Lockard and Choudhari are in agreement with the PIV measurements of Jenkins et al. [15] on a similar high-lift configuration. Figure 9(b), displays contours of the turbulent kinetic energy in the slat cove. Besides the magnitude in the shear layer, they are generally in good agreement with the results published by Deck [21], Lockard and Choudhari [8] and Jenkins et al. [15].

To further highlight the superiority of Case2 over Case1 in terms of scale resolving capabilities, the ratio 


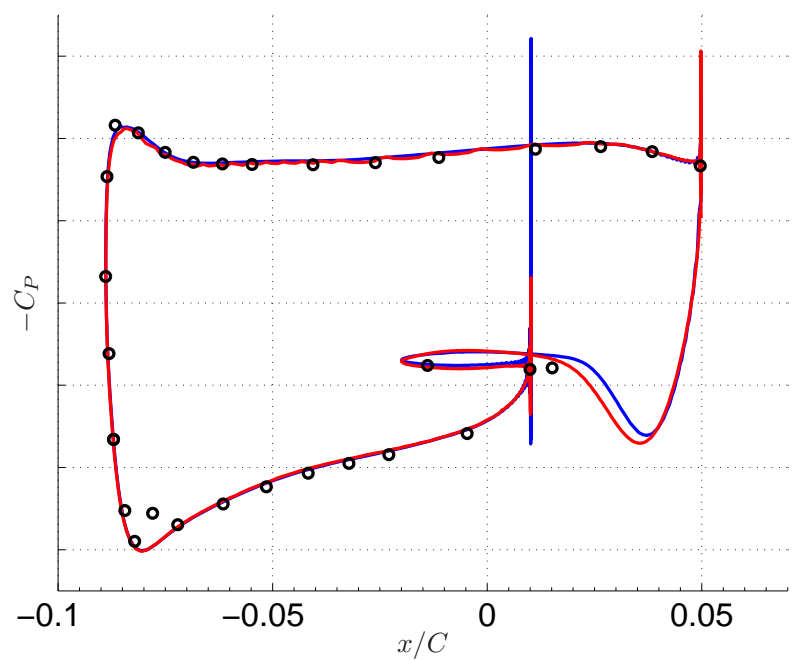

(a) slat

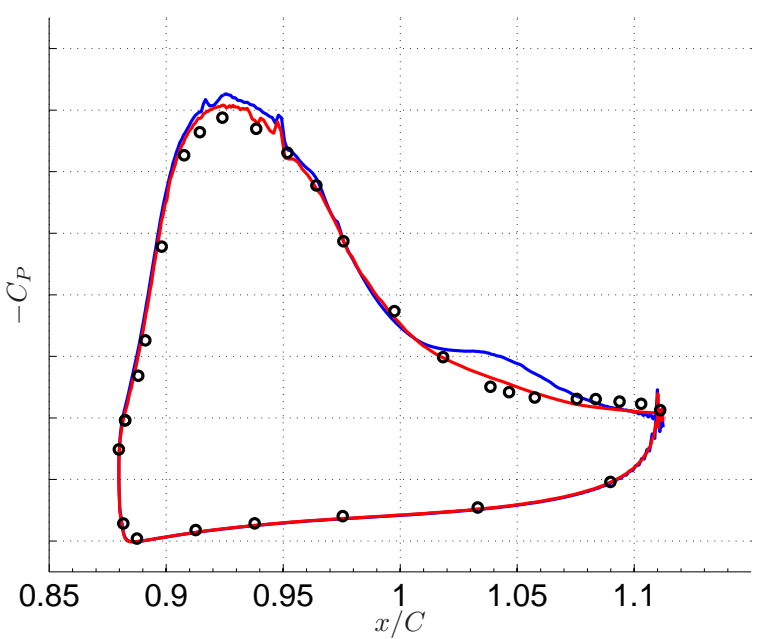

(b) flap

Figure 5: Mean pressure distribution around slat and flap. $\Delta C_{P}=1$

of turbulent viscosity to molecular viscosity, $\mu_{t} / \mu$, is depicted in Fig. 10. A lower turbulent viscosity enables the hybrid RANS/LES model to resolve scales more quickly (i.e. the resolved scales are less dampened) and hence leads to a faster onset of shear layer instabilities. Case 2 consistently exhibits lower levels of turbulent viscosity, except for the peak at the beginning of the shear layer.

Mean streamwise velocity profiles are extracted at locations F1 - F4 around the flap and a comparison of Case1, Case2 and Case3 is shown in Fig. 11. At location F1, shortly upstream of the main wing trailing edge, the velocity profiles differ considerably. This is due to the fact that the flow at this position is strongly influenced by the slat wake and its interaction with the attached boundary layer of the main wing. The turbulence resolving simulations yield similar velocity profiles, which are not as full as the RANS profile. The dent in the profile of Case 2 is caused by the interface between the hexahedral near-wall grid and the unstructured tetrahedral grid in the off-wall region. As the effect of this erroneous velocity profile is not evidenced at the next downstream location, it is assumed that the overall accuracy of the simulation is not compromised. At location F2, the profiles look very similar. As a result of the flow acceleration through the gap between the main wing and the flap, very thin boundary layers are present at the leading edge of the flap. This has been predicted very well by all simulations. Case1 yields higher velocities, which is consistent with location F1. The kink in the profiles at $y / C \approx 0.01$ is the velocity deficit due to the wake of the main wing. It is present also at the next downstream location. All three computations yield different profiles with Case1 predicting the largest deficit. At the most downstream profile, the flow has separated from the surface and has created a zone with reversed flow, which is indicated by the negative streamwise velocity. The largest separation is predicted with RANS, whereas the two HYB0 simulations predict a similar separation bubble size, as already expected from the skin friction distribution in Fig. 6(b).

It was noted that in Fig. 11(a), the velocity profiles appear not as full as the one produced by the SST RANS model. A possible cause can be the so-called Modeled Stress Depletion (MSD), which appears due to grid refinement at the trailing edge of the main wing. The grid becomes fine enough to trigger the switch from RANS to LES mode of the HYB0 model and subsequently the eddy viscosity is reduced from RANS levels to SGS levels. This is shown in Fig. $12(\mathrm{~b})$ at a streamwise location of $x / C \approx 0.85$. Alongside with the reduction of eddy viscosity, also the modeled stresses are degrading, which is highlighted in Fig. 12(a). It can also be seen that the resolved stresses are not able to instantly replace the modeled ones. In severe cases this problem can lead to the so-called Grid Induced Separation (GIS), which has been investigated by Menter [24, 43]. However, in the present case, this behavior is not necessarily undesirable, as the HYB0 model will have switched to LES mode already, when the trailing edge is reached. This should, in principle, limit the delay in the formation of shear layer instabilities. Furthermore, the skin friction plot in Fig. 6(a) reveals that no premature separation occurs at the suction side of the main wing at $x / C \approx 0.85$.

Data of spanwise two-point correlations at three locations in the slat cove region is presented in Fig. 13. The locations P1 - P3 are given in Fig. 7(a) and are situated directly downstream of the slat cusp, near the free shear layer and in the gap between slat and wing, respectively. In a previous study [36], the impact of 


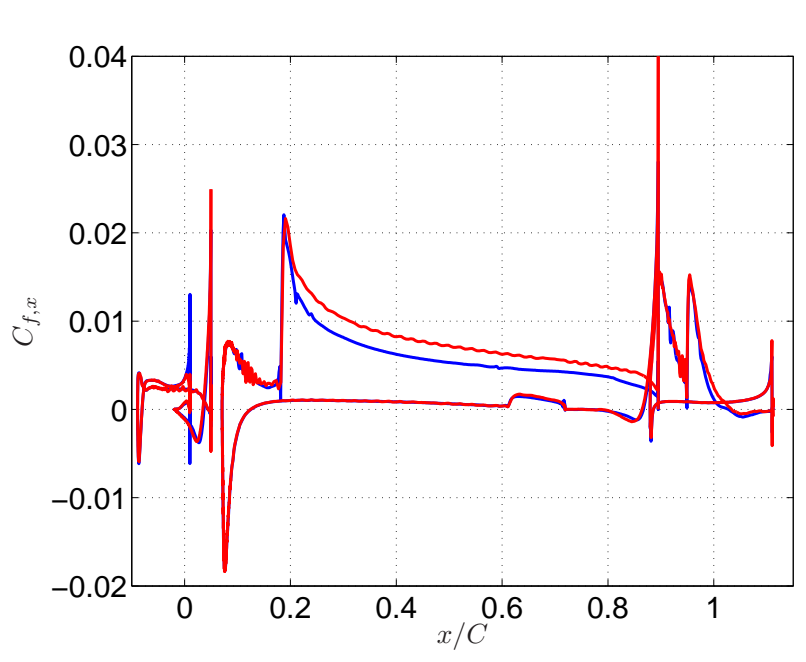

(a) all elements

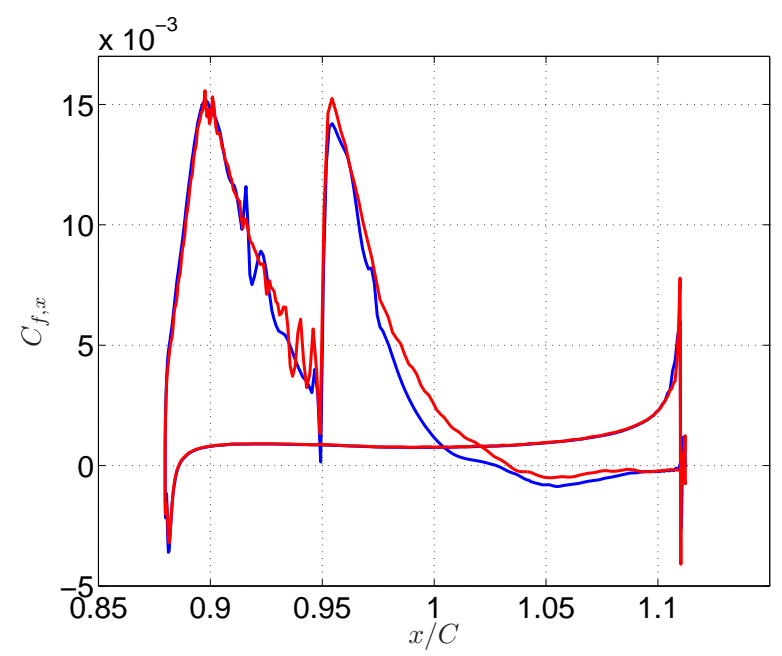

(b) flap

Figure 6: $x$-component of the mean skin friction coefficient, $C_{f, x}$, for all elements (a) and the flap only (b). : Case1, : Case2.

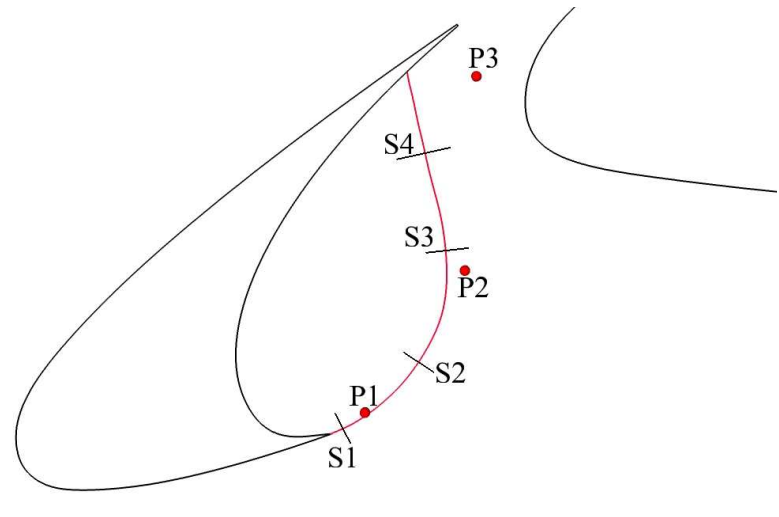

(a) slat

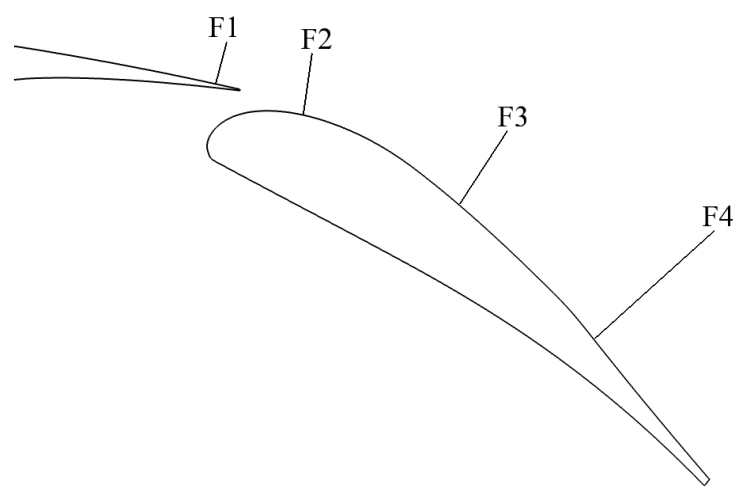

(b) flap

Figure 7: Locations for mean data extraction. At the slat (a), S1 - S4 are cross-sections of the shear layer, P1 - P3 are lines in spanwise direction for plotting the two-point correlations and the red line along the shear layer is a spline, at which data was extracted. At the flap (b), F1 - F4 are the locations of the velocity profiles.

the spanwise domain extent was investigated and it was found that even with a spanwise extent of $0.16 C$, the spatial correlations were strong. This was blamed partly on the grid resolution in the $x-y$ plane and partly on the sampling locations for the two-point correlations themselves, which are placed in regions of strong flow acceleration in which the flow might undergo relaminarization. Here, however, the sampling locations are kept the same. Note that also the spanwise grid resolution is the same in Case1 and Case2. Figure 13 reveals that the two-point correlations are dropping considerably faster and to lower values within the domain for Case2. One can clearly see the effect of the grid resolution in the $x-y$ plane on the flow in spanwise direction. Using the finer grid of Case2, it is possible to resolve smaller structures, which help to destroy the large structures that were found in Case1 (not presented here). The spanwise domain extent can be considered sufficient for the computation in Case2. Nevertheless, the two-point correlation of the pressure fluctuations is of some concern.

The markers on the lines represent the spanwise grid resolution. It can be observed that, if the two-point correlations drop to 0 , they do so within at least 7 cells. This is a useful piece of information, as it shows that at least 7 spanwise cells are used in order to resolve the largest scales. Davidson [44] recommends a minimum of 8 cells to resolve the largest scales in a coarse LES. Hence, the spanwise grid resolution can be deemed borderline for the present study. 


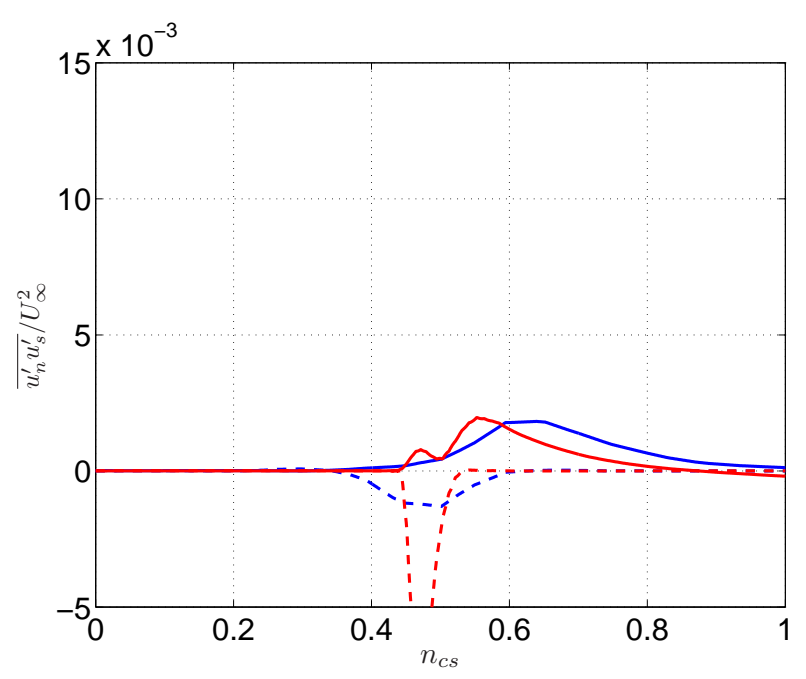

(a) Location S1

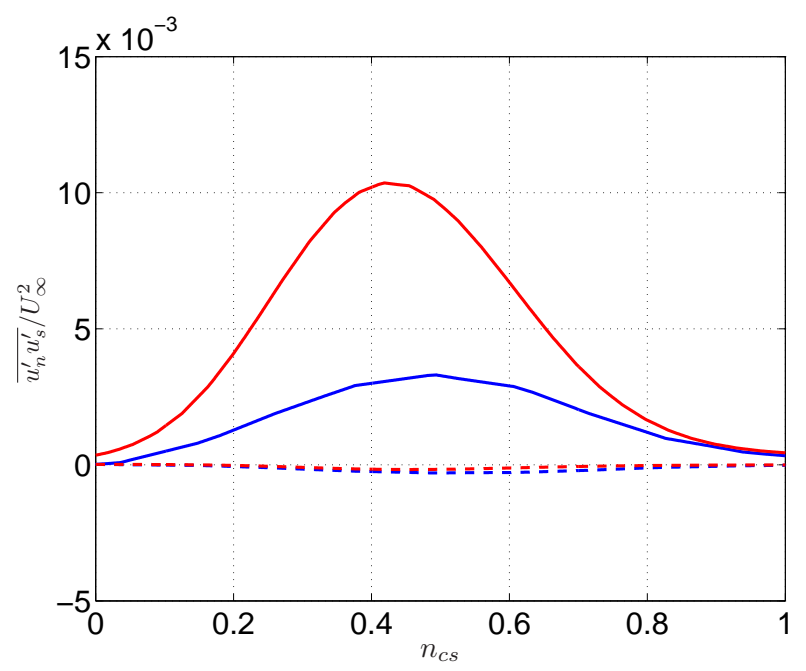

(c) Location S3

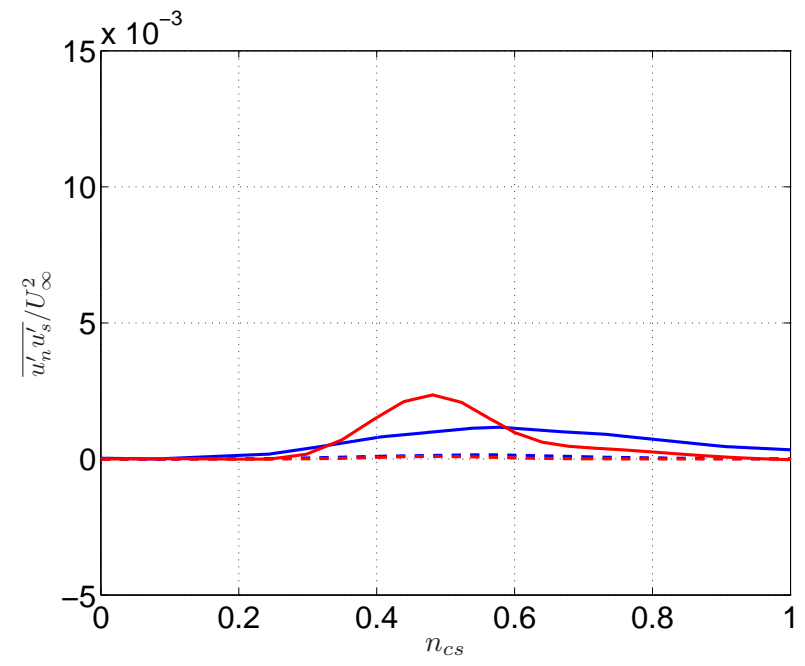

(b) Location S2

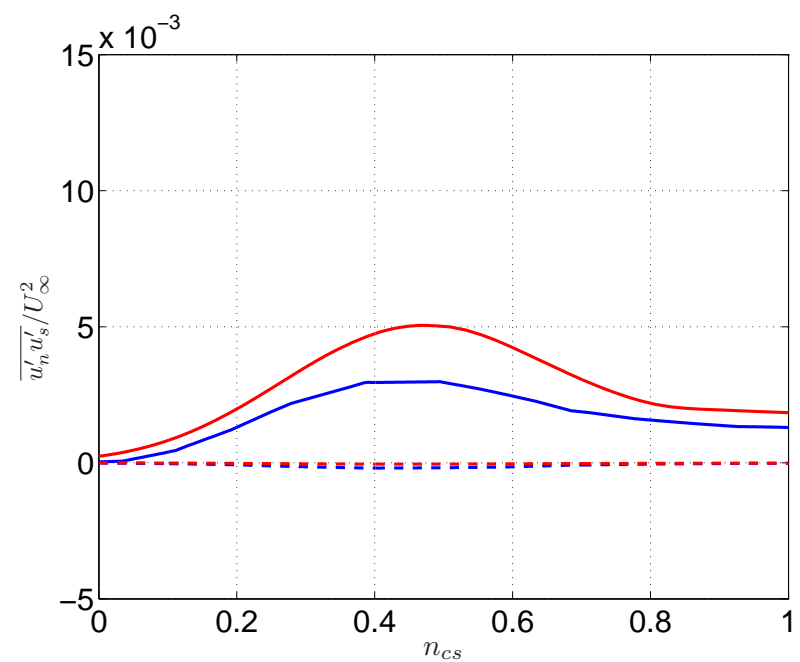

(d) Location S4

Figure 8: Resolved (solid lines) and modeled (dashed lines) shear stress, $\overline{u_{n}^{\prime} u_{s}^{\prime}}$, where $n$ and $s$ denote the normal and tangential direction to the shear layer. Case1, : Case2. $n_{c s}$ denotes the coordinate along the shear layer cross-section.

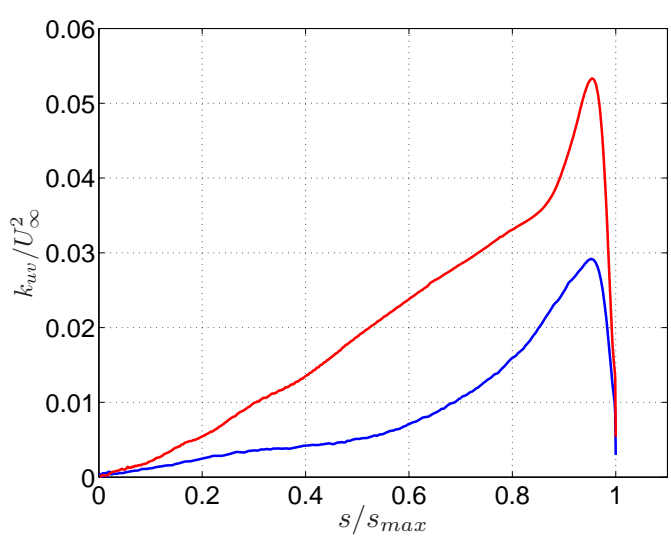

(a)

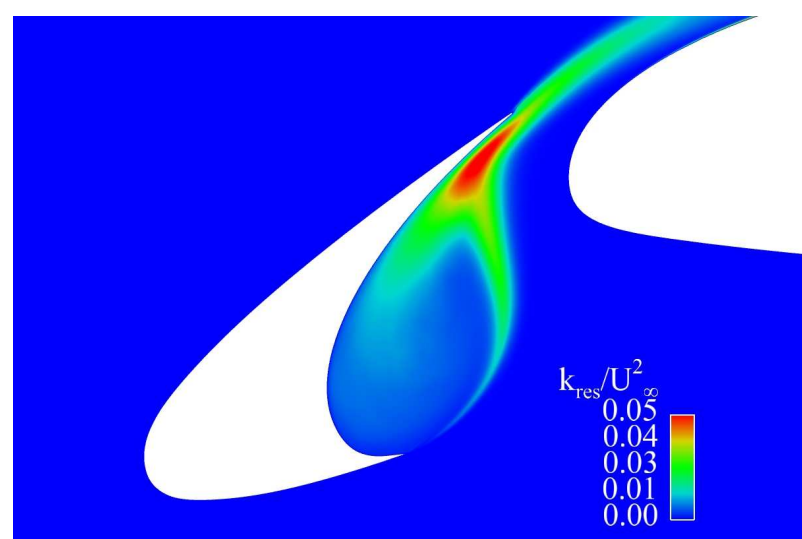

(b)

Figure 9: Resolved turbulent kinetic energy in the slat cove. a) Development of $k_{u v}$ along the shear layer. - Case1, — : Case2. $s / s_{\max }$ denotes the position along the shear layer from the slat cusp. b) Contours of $k_{u v}$ for Case2. 


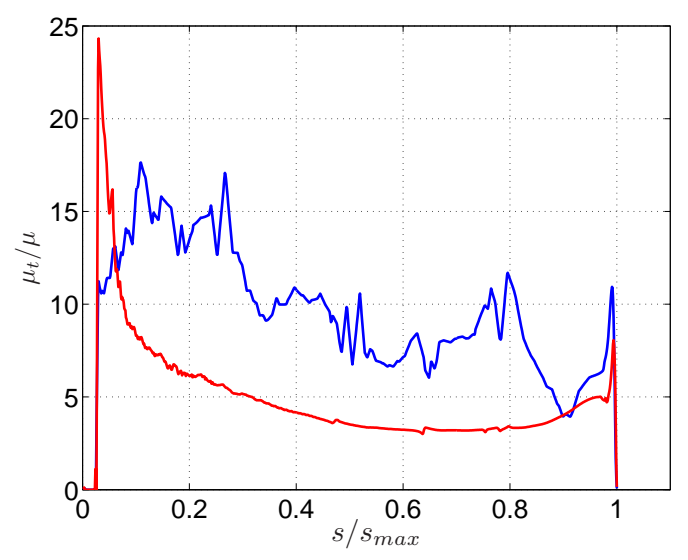

(a)

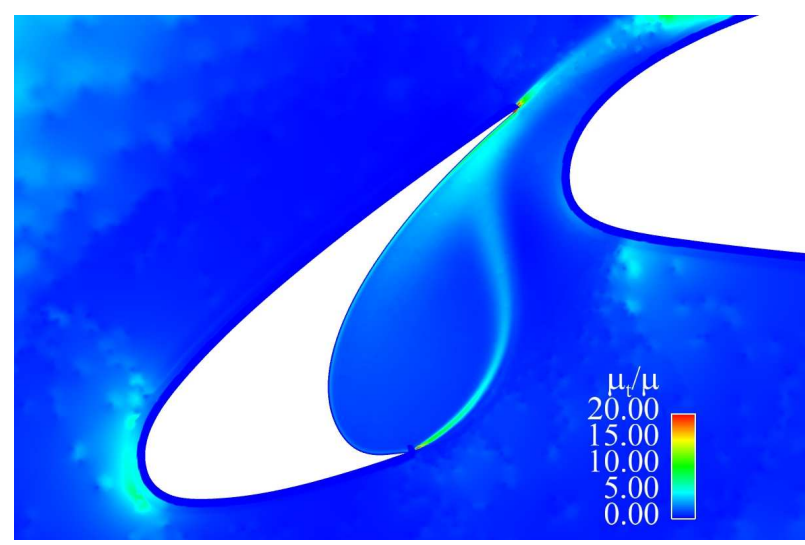

(b)

Figure 10: Viscosity ratio in the slat cove. a) Development of $\mu_{t} / \mu$ along the shear layer.

Case1, Case2.

Case2. $s / s_{\max }$ denotes the position along the shear layer from the slat cusp. b) Contours of $\mu_{t} / \mu$ for

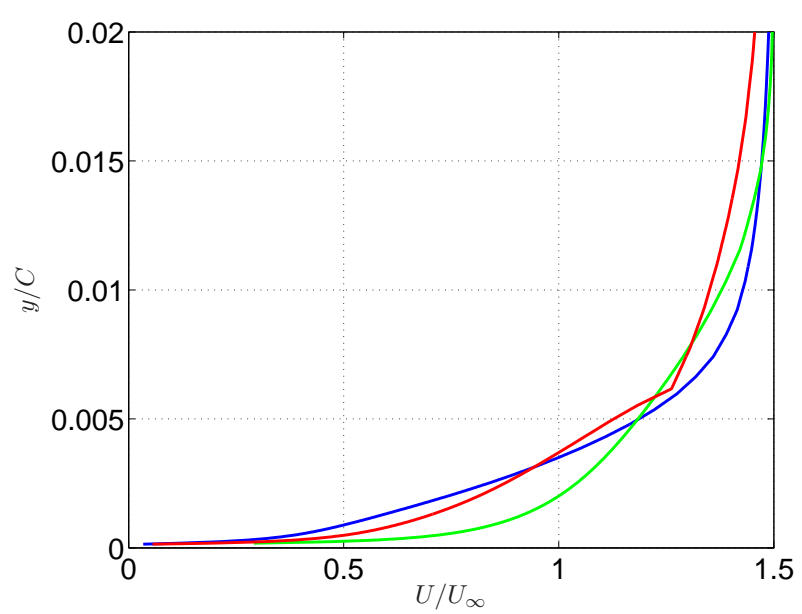

(a) Location F1

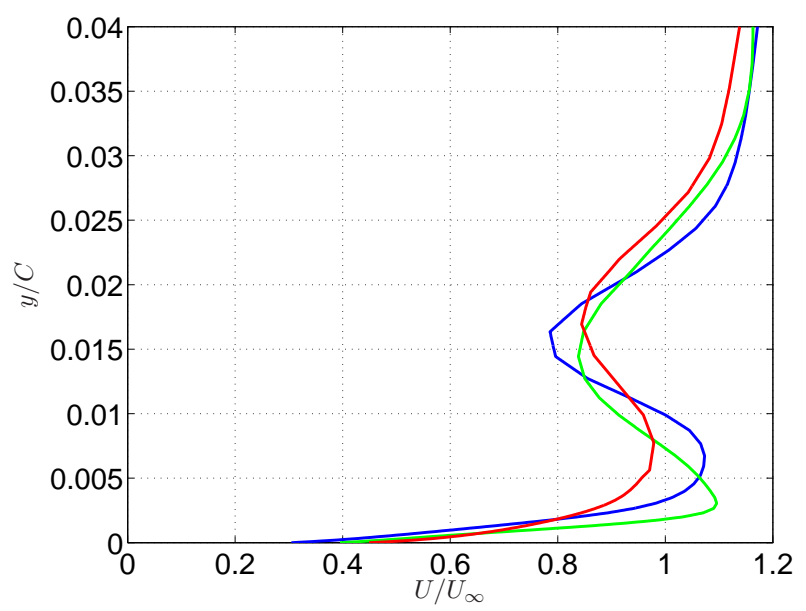

(c) Location F3

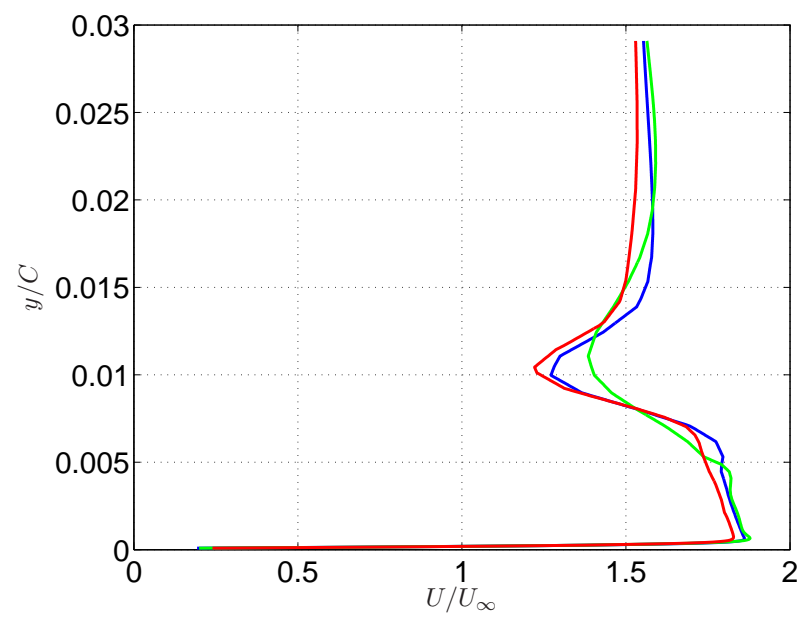

(b) Location F2

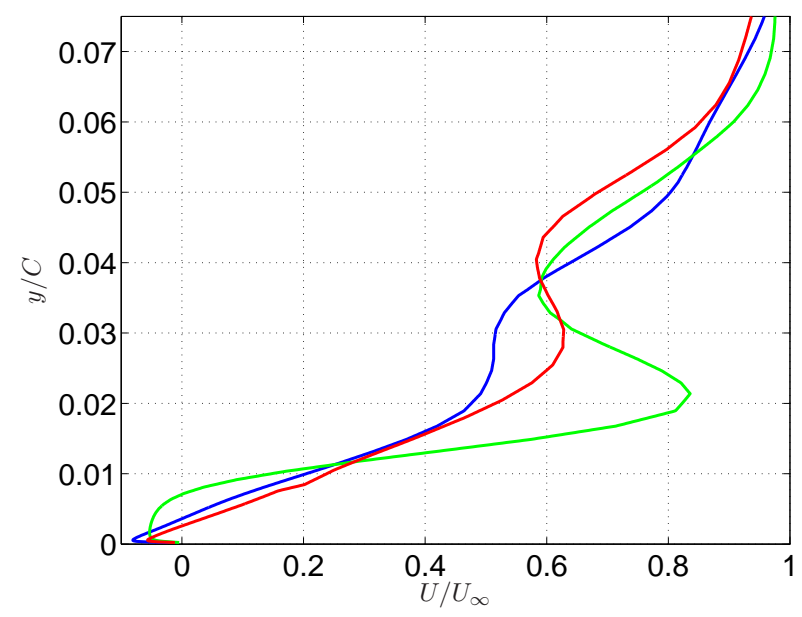

(d) Location F4

Figure 11: Profiles of normalized streamwise velocity, $\frac{|U|}{U_{\infty}}$. Case1, Case2, Case3. 


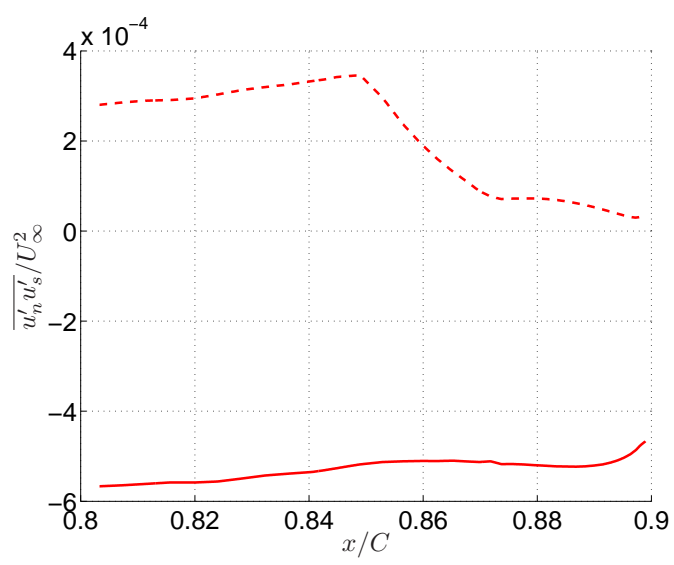

(a)

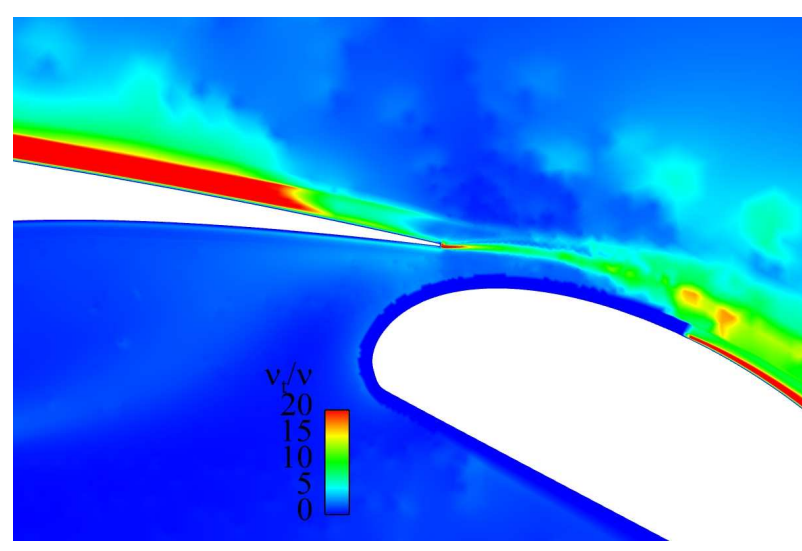

(b)

Figure 12: Modeled stress depletion on the trailing edge of the main wing for Case2. a) Development of modeled and resolved shear stress along a grid line near the trailing edge (distance to the wall: $y / C \approx 0.0045$ ).

resolved, -------: modeled. b) Contours of $\nu_{t} / \nu$ showing the sudden reduction of eddy viscosity towards the trailing edge.

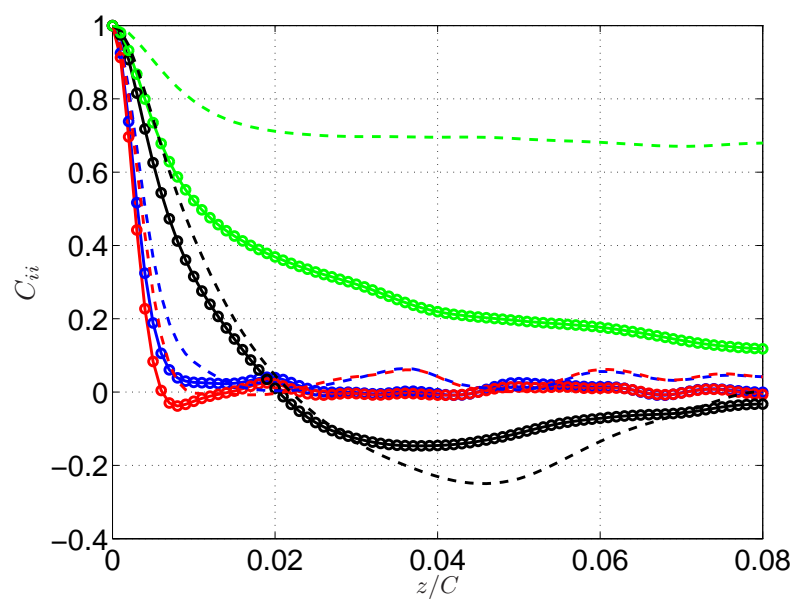

(a) Location P1

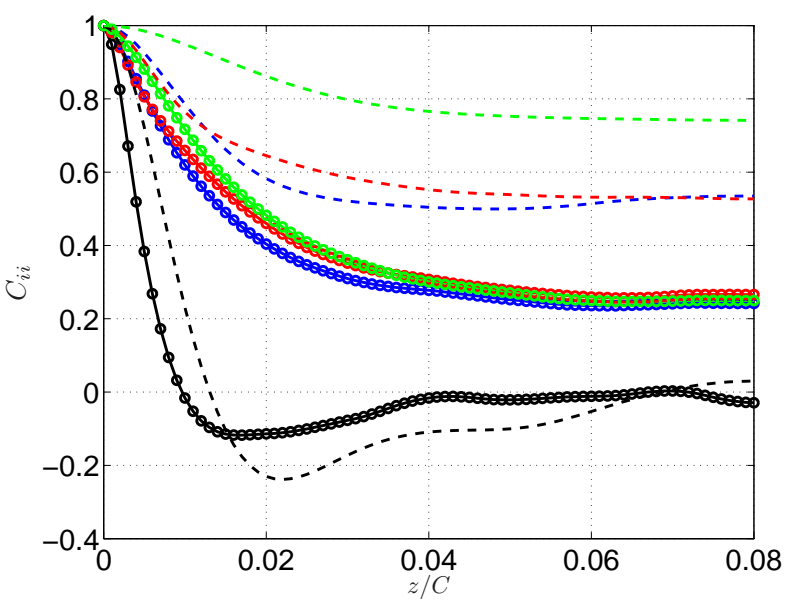

(b) Location P2

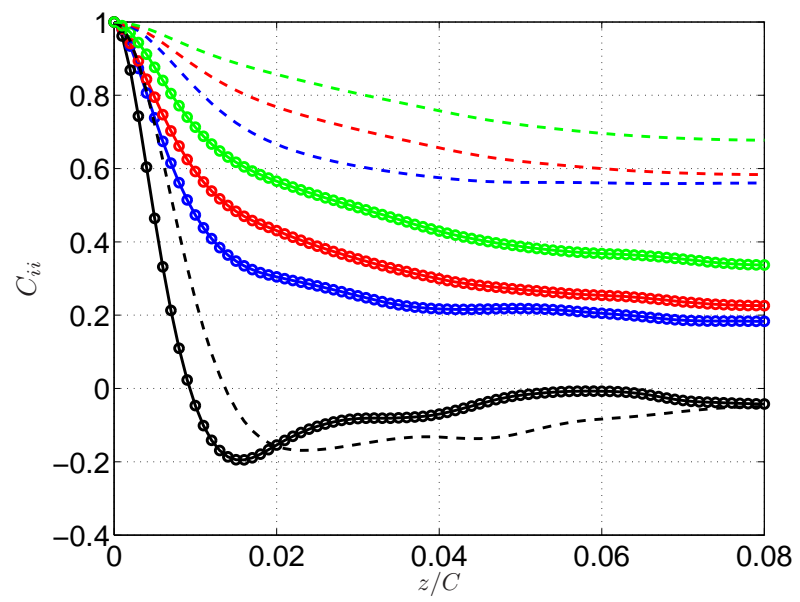

(c) Location P3

Figure 13: Spanwise two-point correlations in the slat cove region; dashed lines: Case1; solid lines: Case2, : $C_{\text {uu,norm }}, \longrightarrow$ : $C_{v v, \text { norm }}, \longrightarrow$ : $C_{w w, n o r m}$ $C_{p p, n o r m}$. Markers on the lines indicate the spanwise resolution. 


\subsection{Aeroacoustic analysis}

As only a small spanwise section of the high-lift wing is investigated here, the radiated noise levels are rather low. This makes it unnecessary to give absolute noise levels and hence only the spacing is indicated on the $y$-axis (or $r$-axis in case of a polar plot). The noise radiation is computed at 72 observers located in a circle of radius $300 C$ around the airfoil. In the following, both the Sound Pressure Level (SPL) and the OverAll Sound Pressure Level (OASPL) for the different acoustic analogies will be presented. The Sound Pressure Level and the OASPL are defined as

$$
\begin{aligned}
S P L & =20 \log _{10}\left(\frac{p^{\prime}}{p_{\text {ref }}}\right) \\
O A S P L & =20 \log _{10}\left(\frac{p_{\text {rms }}}{p_{\text {ref }}}\right)
\end{aligned}
$$

with $p_{\text {ref }}$ being the reference pressure. In the present case $p_{\text {ref }}=2 \times 10^{-5}[P a]$ is chosen, which usually is considered the threshold of human hearing. An entire circle around the airfoil is computed so that it becomes possible to determine, in which direction the sound is mainly radiated. Figure 14 shows the polar coordinate system used and indicates that the upstream and downstream directions of the airfoil are aligned with the coordinates $\theta=0^{\circ}$ and $\theta=180^{\circ}$, respectively. The upwards and downwards directions become then $\theta=90^{\circ}$ and $\theta=270^{\circ}$, respectively.

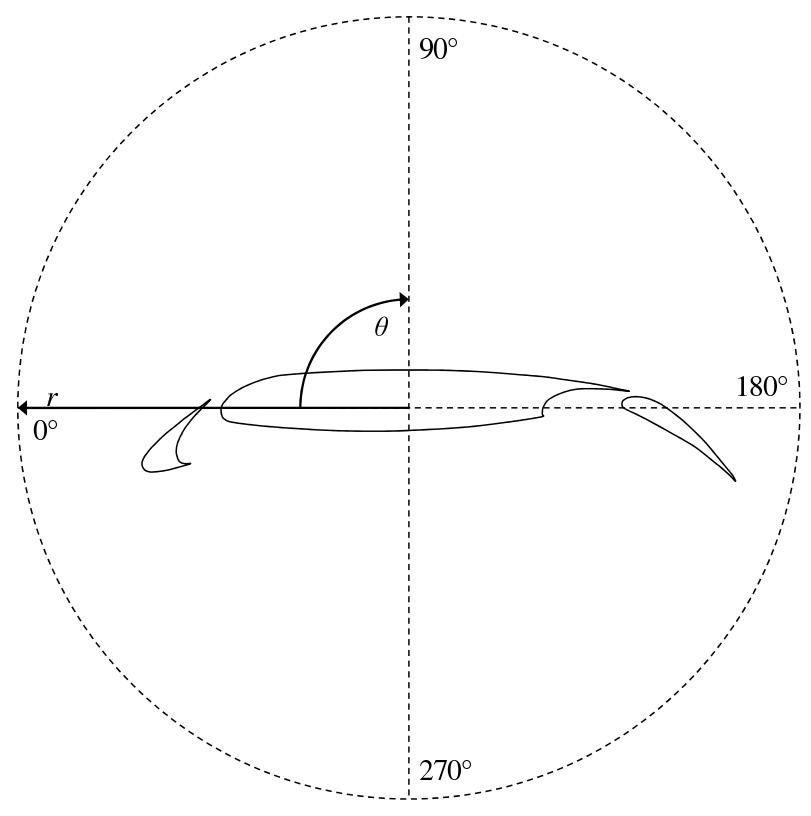

Figure 14: Polar coordinate-system

From a practical point of view, only the sound radiated in the downward direction, i.e. $\theta=270^{\circ}$, would be of interest, but for completeness and for comparison, all 360 degrees are included in the directivity plots. Four observer locations at $\theta=0^{\circ}, \theta=90^{\circ}, \theta=180^{\circ}$ and $\theta=270^{\circ}$ were chosen for presenting the SPL plots. The SPL is plotted against the Strouhal number, $S t=f C_{s} / U_{\infty}$, based on the slat chord, $C_{s}$. Please note that in principle the result at $\theta=180^{\circ}$ should be incorrect, as the downstream part of the integral surface is kept open.

\subsubsection{Analysis using the Kirchhoff surface integral method}

The SPL obtained with the Kirchhoff method are depicted in Fig. 15(a)-(d). Included in the plots are both the SPL due to the components of the fluctuating pressure, $p_{i, K}^{\prime}$, as well as the SPL due to their sum, $p_{\text {total,K}}^{\prime}$. In general a broad banded spectrum is obtained, which peaks at low Strouhal numbers for $S t<3$. Moreover, at Strouhal numbers in the range of $S t \approx 1-5$ several narrow banded tonal peaks are present. Several other research groups also found the peak SPL levels around Strouhal numbers of 1-3 and confirm the presence of tonal peaks in the low-frequency range [3,8,11]. The downward direction exhibits the strongest tonal peak at $S t \approx 2.8$ with adjacent peaks at $S t \approx 0.95, S t \approx 1.9, S t \approx 3.6$ and $S t \approx 4.5$. In comparison to previous 
near-field noise studies on this geometry [36], there are more tonal peaks present, which are shifted towards slightly higher frequencies. At $S t \approx 40$, the spectra exhibit a broad banded peak. It is most obvious in the upstream and downstream directions, but can also be observed in the other two directions. A similar peak has previously been reported by several authors, for example, Choudhari et al. [45] and Khorrami [46]. It is the major source of high-frequency slat noise and is believed to stem from vortex shedding behind the blunt trailing edge of the slat. Further evidence for this hypothesis can be provided, when plotting the spectra in Fig. 15 versus a Strouhal number based on the trailing edge thickness of the slat, $S t_{T E}$. In that case, the peak appears at $S t_{T E} \approx 0.12$, which is of the same order of magnitude as the frequency commonly associated with vortex shedding behind circular cylinders, $S t_{c y l} \approx 0.2$ [47]. Hence, the peak is indeed related to vortex shedding behind the blunt trailing edge of the slat. In previous research on another three-element airfoil at higher Reynolds numbers [7,14], this peak appears at higher frequencies and is often shown to be the major peak in the spectrum, which is not the case here. These differences can be attributed to the disparity of the investigated airfoils along with their test conditions.

At all four positions, $p_{1, K}^{\prime}$ gives by far the smallest amplitude and does not significantly contribute to the total noise $p_{\text {total }, K}^{\prime}$. It is hence excluded from the plots in Fig. 15. Both, $p_{2, K}^{\prime}$ and $p_{3, K}^{\prime}$ follow the total noise quite well. However, at low Strouhal numbers below $S t \approx 2, p_{3, K}$ yields considerably lower amplitudes than $p_{2, K}^{\prime}$, which will have a considerable impact on the OASPL meaning that $p_{2, K}^{\prime}$ will contribute most to the total sound pressure, $p_{\text {total }, K}^{\prime}$, at the observers.

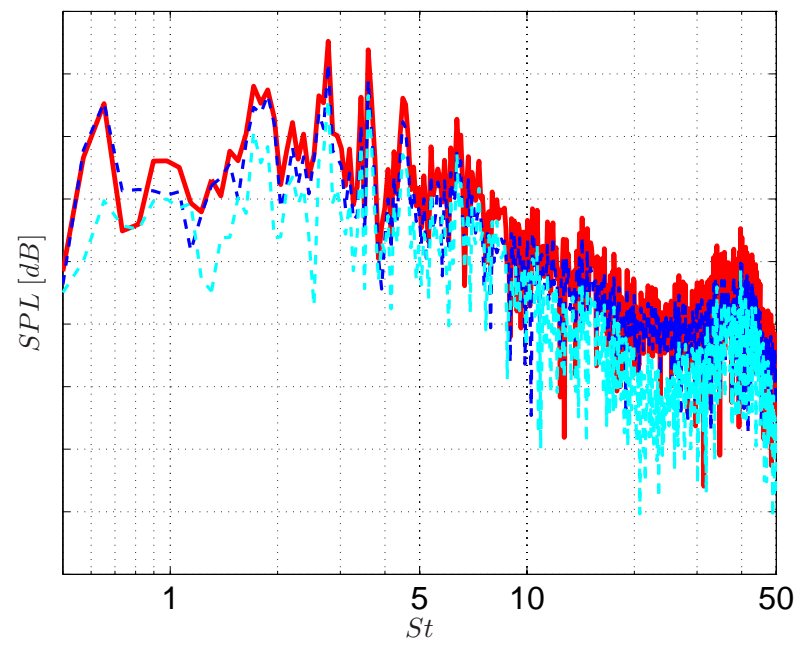

(a) $\theta=0^{\circ}$

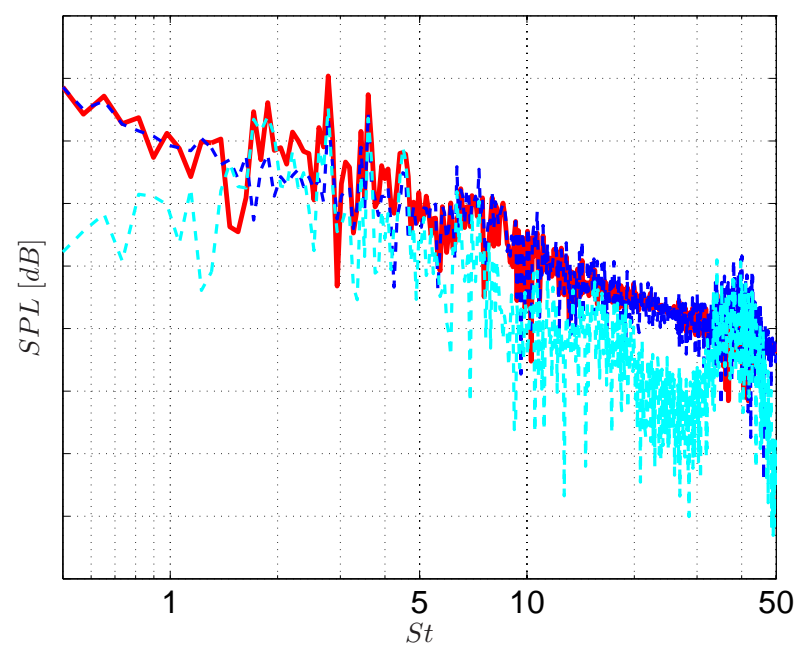

(c) $\theta=180^{\circ}$

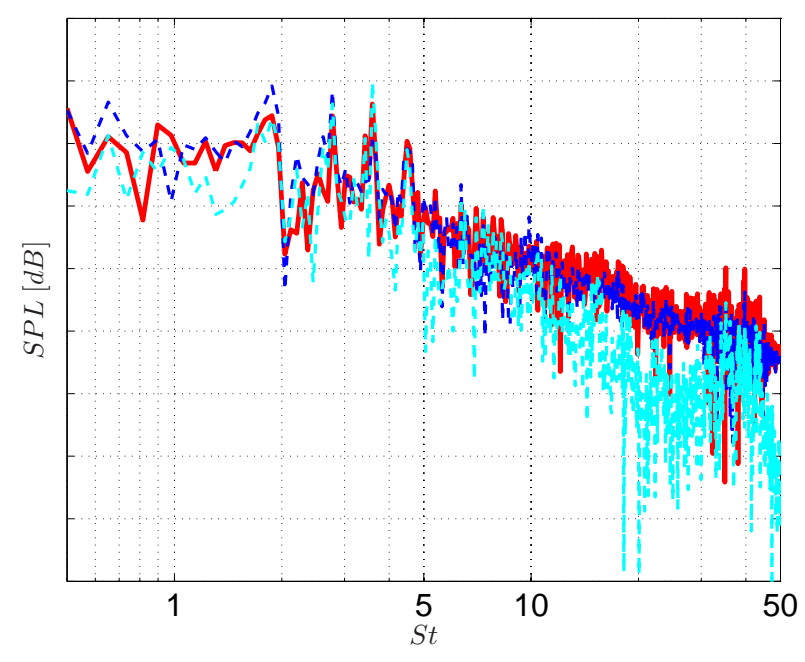

(b) $\theta=90^{\circ}$

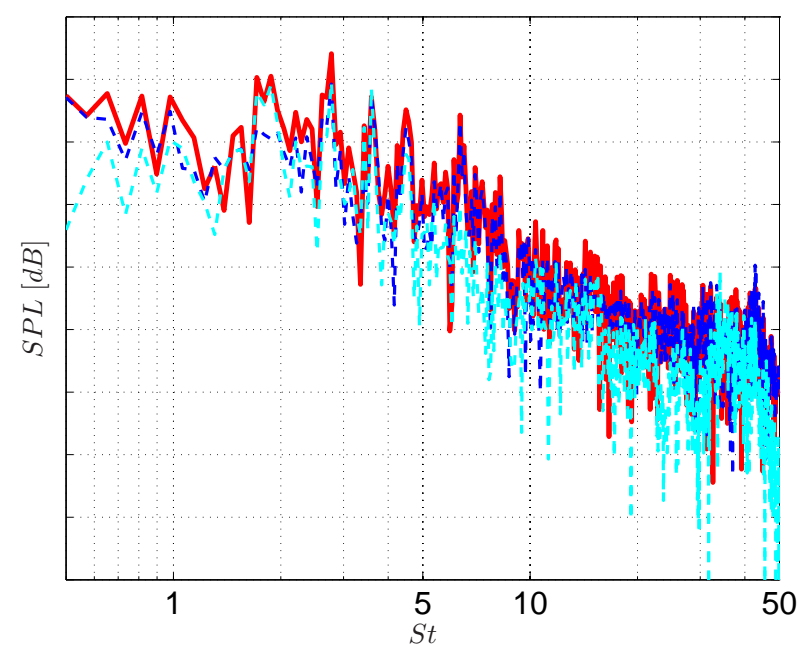

(d) $\theta=270^{\circ}$

Figure 15: SPL for the Kirchhoff integral method. $10[d B]$.

It is displayed in Fig. 16(a) that the Kirchhoff method predicts the total sound to behave like a monopole. 
As expected, the largest contributor to the total noise is term 2 (Eq. (3b)). Yao and co-workers [10] show that the noise due to term 3 behaves like a dipole with radiation roughly in the $\theta=100^{\circ}$ and $\theta=280^{\circ}$ directions. Only weak dipole trends with radiation maxima roughly in the $\theta=80^{\circ}$ and $\theta=240^{\circ}$ directions can be observed here, but it is likely that the present high-lift configuration with an additional slat behaves differently, as the one investigated by Yao and co-workers. The shaky behavior of $p_{3, K}^{\prime}$ in the downstream direction might be caused by the fact that the integral surface is kept open in that direction. It is, however, evident that the source term directly connected with the pressure fluctuations, $p_{1, K}^{\prime}$, exhibits a dipole emission, but at the same time does not play an important role in the overall sound. A look at Eq. (3a) reveals that $p_{1, K}^{\prime}$ can only be important for near-field noise, as it decreases with the square of the observer distance. Hence, it is not a surprising result that the contribution of term 1 is negligible at the present observer distance.

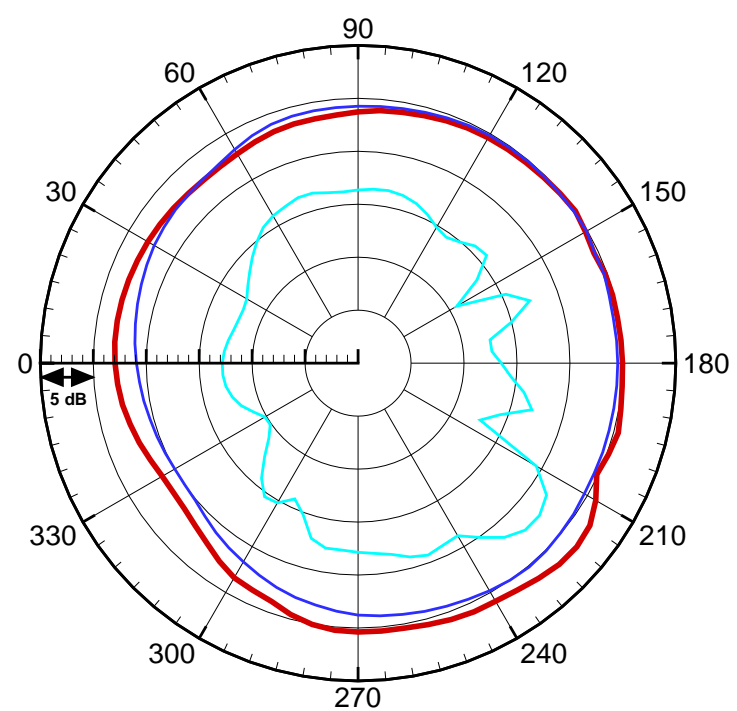

(a)

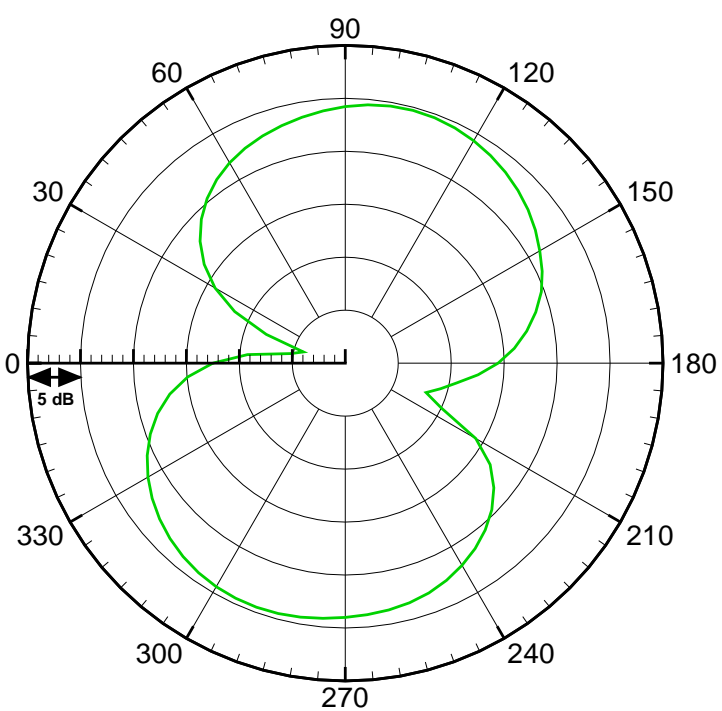

(b)

Figure 16: OASPL for the Kirchhoff integral method. In b), $p_{1, K}^{\prime}$ is depicted, as it is much smaller than the other terms (different scaling in b)). — : $p_{\text {total }, K}^{\prime}, \longrightarrow: p_{1, K}^{\prime}, \longrightarrow: p_{2, K}^{\prime}, \longleftarrow: p_{3, K}^{\prime}$.

\subsubsection{Analysis using the FWH method}

Figure 17 presents the SPL obtained with the FWH method on the permeable and stationary surface. The contributions of the three surface integrals defined by Eq. (6) as well as the total sound pressure are included in the plots. Similar to the results with the Kirchhoff integral method, a broad banded spectrum with narrow banded tonal peaks is shown and also the previously mentioned high-frequency peak at $S t \approx 40$ can be identified. The sound pressure due to the second term, $p_{2, F}^{\prime}$ yields consistently the lowest levels with the weakest signal in the upwards and downwards directions. Recall that $p_{2, F}^{\prime}$ is related to the momentum perturbations at the surface. One can then conclude that the momentum perturbations at the surface location are in general low. It can be seen that $p_{1, F}^{\prime}$ closely follows the total sound pressure $p_{\text {total,F}}^{\prime}$, but that it does not represent the high-frequency peak. In the reverse, this means that the high-frequency peak is associated solely with $p_{3, F}^{\prime}$ and hence can be connected to the perturbations of mass through the surface, as made clear by Eq. (6c).

The directivity plots obtained by the FWH analogy are displayed in Fig. 18. They do not show a clear trend towards one of the acoustical source types, but can rather be described as a superposition of different sources. Three flat radiation maxima can be found for $\theta \approx 30^{\circ}, \theta \approx 180^{\circ}$ and $\theta \approx 240^{\circ}$, which correlates well with Lockard's observations [8] for a similar high-lift airfoil using the FWH analogy on a stationary, permeable surface and an observer distance of $10 \mathrm{C}$. In Fig. 17, $p_{2, F}^{\prime}$ was found to be the smallest with the lowest SPL levels in the upwards and downwards directions. Figure $18(\mathrm{~b})$ makes clear that $p_{2, F}^{\prime}$ radiates like a dipole in the up- and downstream directions. Subsequently, the noise contribution due to the momentum perturbations is not of practical importance in terms of noise reduction. When plotted in a different scaling (not shown here), $p_{1, F}^{\prime}$ exhibits an emission of dipole nature with maxima in the upwards and downwards directions. Thus, $p_{1, F}^{\prime}$ and $p_{2, F}^{\prime}$ are radiating noise roughly orthogonal to each other. Similar behavior was experienced by Yao et al. [10] for high-lift wings without a leading edge slat. In the range of $\theta \approx 210^{\circ}-300^{\circ}, p_{1, F}^{\prime}$ is the dominant source of noise by roughly $2-5[d B]$, whereas in the up- and downstream directions $p_{3, F}^{\prime}$ exceeds $p_{1, F}^{\prime}$ by about $5[d B]$ 


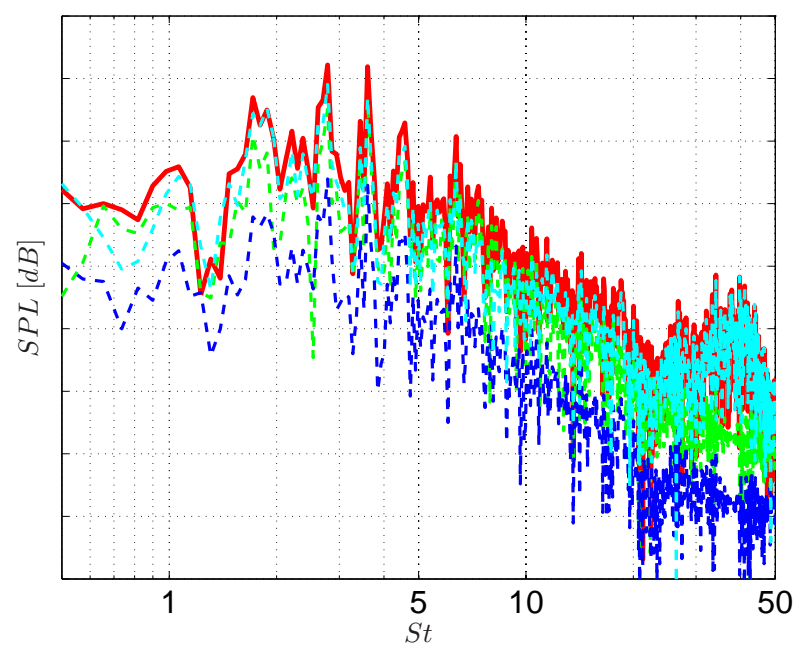

(a) $\theta=0^{\circ}$

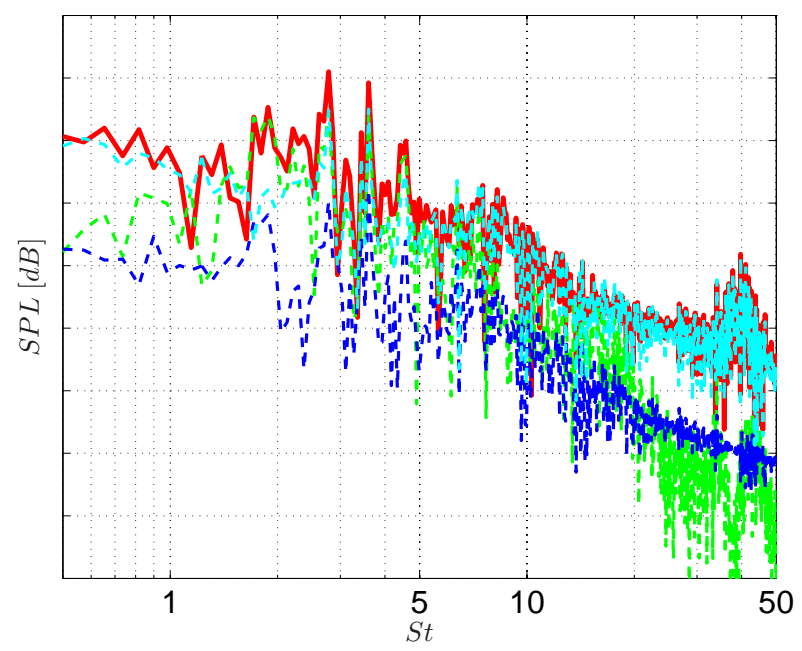

(c) $\theta=180^{\circ}$

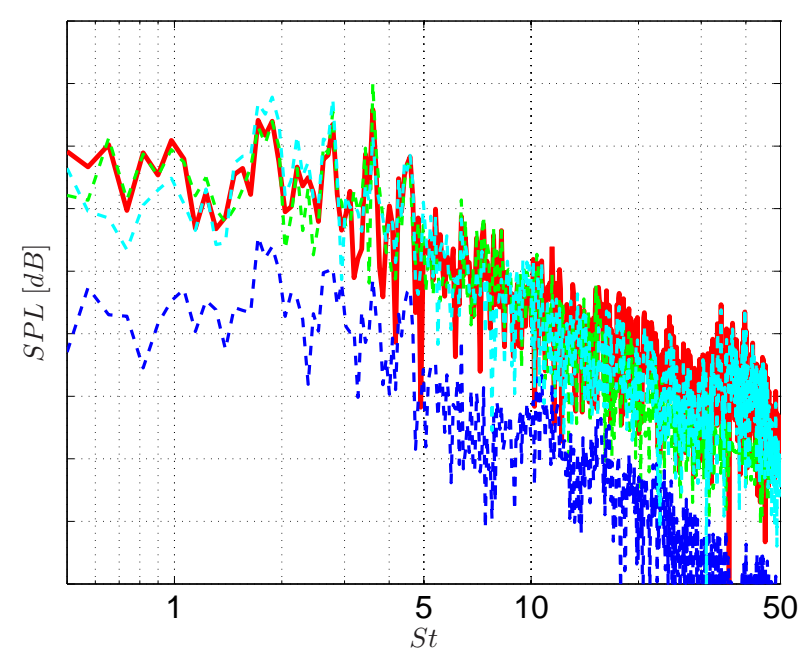

(b) $\theta=90^{\circ}$

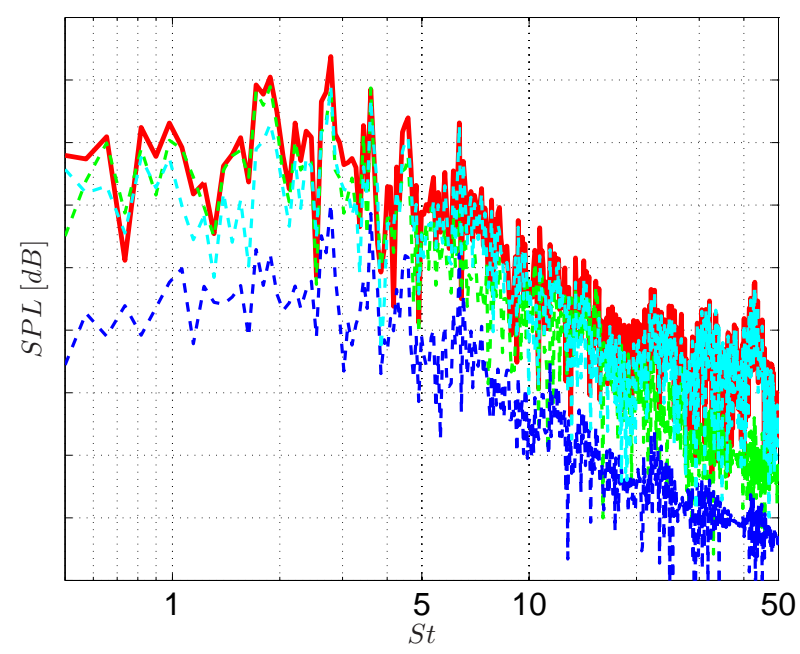

(d) $\theta=270^{\circ}$

Figure 17: SPL obtained with the FWH method. $\triangle S P L=10[d B]$.

and hence is governing the total noise.

\subsubsection{Analysis using Curle's method}

The Curle method as described earlier, does only account for the pressure fluctuations at stationary, solid surfaces due to the flow. Hence, for the following analysis, the solid walls of the three elements of the airfoil, namely slat, wing and flap, are used. The total sound pressure, $p_{\text {total, } C}^{\prime}$ corresponds in this case to the sum of the pressures of the three elements. This allows to treat the noise radiation of each element in isolation.

The spectra displayed in Fig. 19 also exhibit a broad banded shape for all of the elements and the total noise with peak levels in the range of $S t \approx 1-3$. Besides the fact that the narrow banded tonal peaks are present, the absence of the high-frequency peak is most obvious. As stated earlier, the high-frequency peak is associated to the perturbations of mass through the permeable surface, which is not accounted for in Curle's method. The slat claims the highest SPL amplitudes at all four presented observer locations. It has been widely acknowledged that the slat indeed is the major contributor to noise due to the high-lift system [2,3,11-13]. The spectra obtained for the flap tend to decay faster at Strouhal numbers beyond $S t \approx 20$. In the downwards direction, the SPL plots indicate that the wing and flap do not contribute to the total noise as much as the slat.

Figure 20 depicts the directivity plots for the three elements together with the OASPL of the total noise. The total noise has its radiation maxima in directions $\theta \approx 15^{\circ}$ and $\theta \approx 230^{\circ}$. It is evident that the slat radiates as a dipole and is the main contributor to the total noise. Exactly as the total noise, the slat predominantly 


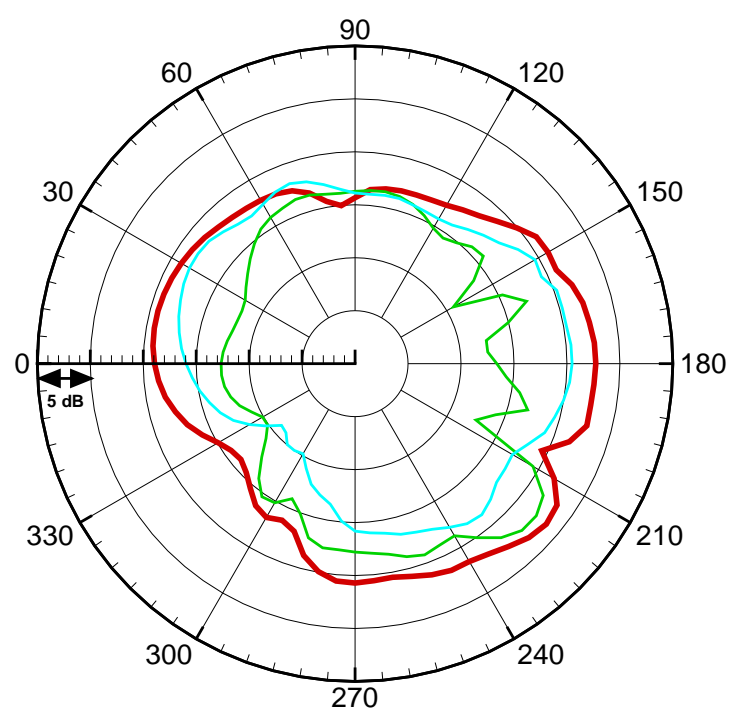

(a)

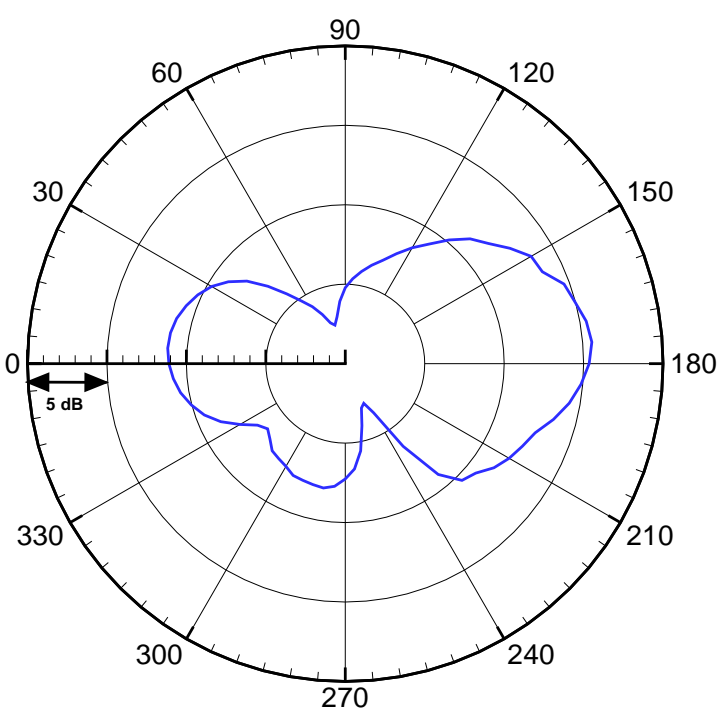

(b)

Figure 18: OASPL for the FWH method. In b), $p_{2, F}^{\prime}$ is depicted, as it is smaller than the other terms. Note that the maximum of b) corresponds to the 3rd lowest level in a). $-: p_{\text {total }, F}^{\prime}, \longrightarrow: p_{1, F}^{\prime}, \longrightarrow: p_{2, F}^{\prime}$, $: p_{3, F}^{\prime}$.

radiates into the upper forward $\operatorname{arc}\left(\theta \approx 15^{\circ}\right)$ and in the lower rearward direction $\left(\theta \approx 230^{\circ}\right)$. The wing shows no distinct behavior, but rather a superposition of different sources. The strongest emission, however, is found in the direction of $\theta \approx 150^{\circ}$. Except in that and in the opposite direction, the wing emits much lower levels than the slat and hence does not contribute considerably to the total sound pressure. Even though of low intensity, the flap exhibits an almost perfect dipole emission in the upper rearward direction $\left(\theta \approx 135^{\circ}\right)$ and the lower forward region $\left(\theta \approx 315^{\circ}\right)$. Recall that the slat and flap are deflected by $28.8^{\circ}$ and $38.3^{\circ}$ with respect to the $\theta$-axis. It then becomes obvious that both slat and flap are emitting orthogonally to their own orientation. The wing could possibly be another superposition of two dipoles directed almost orthogonally to each other.

\subsubsection{Comparison of the acoustic analogies}

As in the previous sections the results obtained with the different acoustic analogies were generally similar, but deviations from each other were not negligible, a detailed comparison between the methods is carried out here.

Firstly, the Sound Pressure Levels obtained by the three acoustic analogies are compared in Fig. 21. The SPL of the three methods show similar spectra with the tonal peaks predicted at almost identical frequencies. However, Curle's method yields the lowest amplitudes, whereas the Kirchhoff and FWH methods yield amplitudes that are more similar to each other. In the downstream direction, the Curle method shows a faster decay than the other two methods. Table 2 summarizes the frequencies of the five tonal peaks indicated in Fig. 21(d). Curle's method predicts the tonal peaks at slightly lower Strouhal numbers as Kirchhoff's method, whereas the FWH method predicts slightly higher Strouhal numbers for the same peaks.

Table 2: Strouhal numbers of tonal peaks

\begin{tabular}{cccccc}
\hline \hline & Peak I & Peak II & Peak III & Peak IV & Peak V \\
\cline { 2 - 6 } & St & St & St & St & St \\
\hline Kirchhoff & 1.88 & 2.78 & 3.59 & 4.49 & 6.36 \\
Curle & 1.86 & 2.73 & 3.61 & 4.44 & 6.34 \\
FWH & 1.88 & 2.78 & 3.60 & 4.58 & 6.38 \\
\hline
\end{tabular}

The OASPL of the respective analogies are cross-plotted in Fig. 22. Note, that the scaling has been changed in comparison to the previous OASPL plots in order to emphasize the differences. The Kirchhoff method consistently predicts the highest sound pressure levels and Curle's method consistently predicts the lowest level with the Ffowcs-Williams and Hawkings method in between the two. Deviations between the Curle and the Kirchhoff method are considerable and range between 5-10 $[d B]$. Even compared to the FWH method, Kirchhoff yields at least $4[d B]$ higher levels of sound pressure. The Kirchhoff method yields such high values 


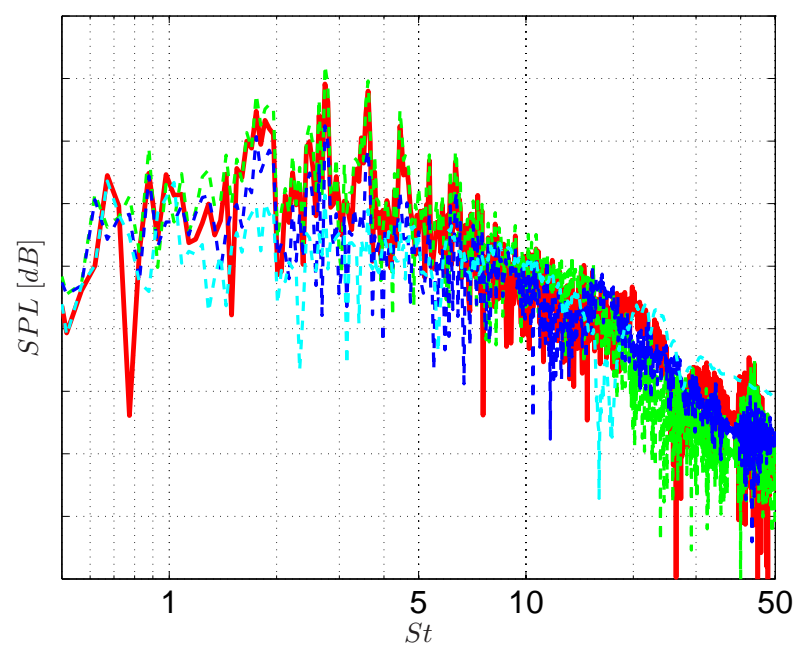

(a) $\theta=0^{\circ}$

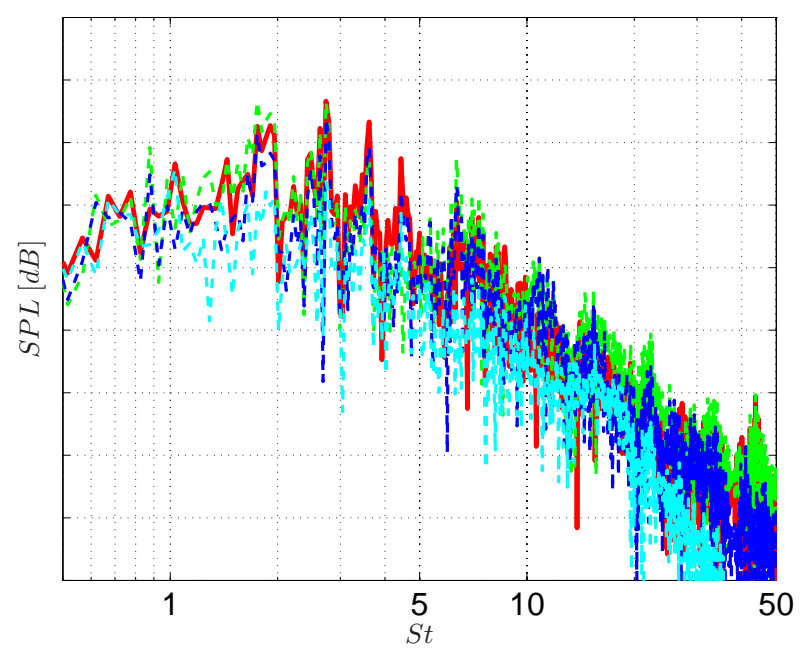

(c) $\theta=180^{\circ}$

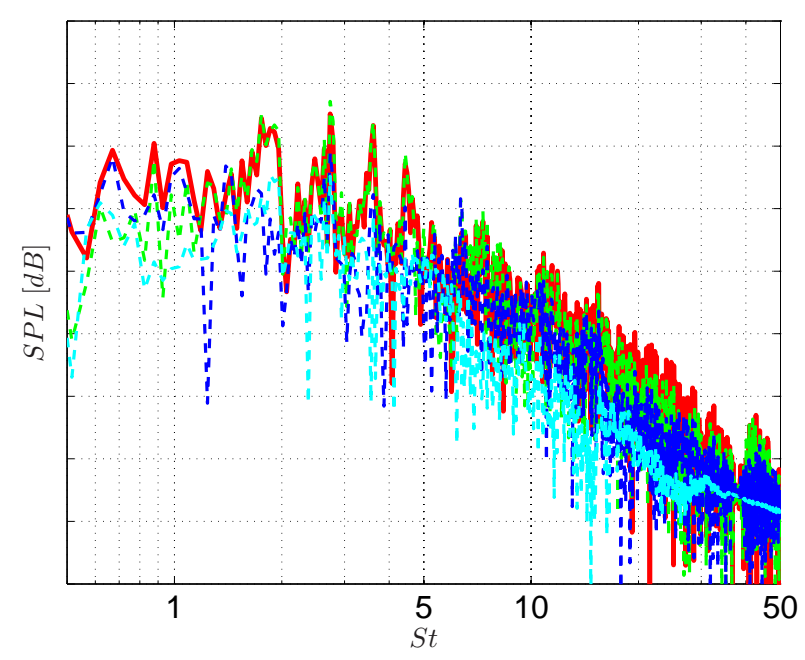

(b) $\theta=90^{\circ}$

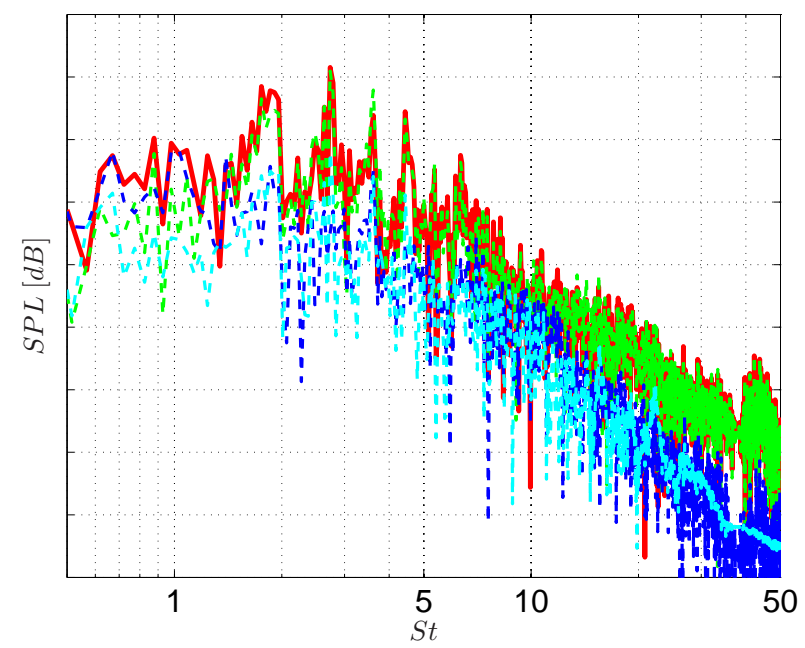

(d) $\theta=270^{\circ}$

Figure 19: SPL obtained with the Curle method. $p_{\text {flap }, C}^{\prime} . \triangle S P L=10[d B]$.

: $p_{\text {total }, C}^{\prime},-------: p_{\text {slat }, C}^{\prime},-------: p_{\text {wing }, C}^{\prime}$,

due to $p_{2, K}^{\prime}$. Excluding this term from the calculation of the total noise results in noise levels comparable to the ones of the FWH and Curle methods (not presented here). The Curle analogy only takes into account the contribution of the pressure fluctuations at the solid walls of the three airfoil elements. In the FWH analogy, also the noise from the turbulence in the flow field is involved, which helps to explain the observed differences between the two methods. Despite the deviations in amplitude, the Curle and FWH methods yield similar radiation patterns, which clearly highlights the alikeness of the two approaches. It was explained earlier that the FWH method for a stationary surface, would be identical to the Curle method, if the solid walls of the airfoil elements were chosen instead of the permeable surface. To further illustrate the similarity of the Curle and FWH methods, $p_{1, F}^{\prime}$ in Figure 17 is not able to represent the high-frequency peak, since it is identical to $p_{\text {total,C }}^{\prime}$ in the Curle method.

Figures 16 and 18 reveal that $p_{3, K}^{\prime}$ and $p_{1, F}^{\prime}$ show almost identical results. By inspection of Eq. (6a) one can see that $p_{1, F}^{\prime}$ is actually identical with the sum of $p_{1, K}^{\prime}$ and $p_{3, K}^{\prime}$ (Eq. (3)). It was shown earlier that $p_{1, K}^{\prime}$ is negligible in the far-field, as it decreases with the square of the observer distance. Hence, it is an expected outcome that $p_{3, K}^{\prime}$ and $p_{1, F}^{\prime}$ yield similar results.

Frequency spectra of the fluctuating pressure for the downward observer location are presented in Fig. 23. Note that on the $x$-axis, the Strouhal number is plotted in logarithmic scale. On the $y$-axis, the Strouhal number multiplied with the power spectral density is plotted in linear scale. The energy content of a range of frequencies is hence characterized by the area under the curve. The tonal peaks at $S t \approx 0.95, S t \approx 1.9$, $S t \approx 2.8, S t \approx 3.6$ and $S t \approx 4.5$ can be recognized. By far the highest peak is located at $S t \approx 2.8$. One can 


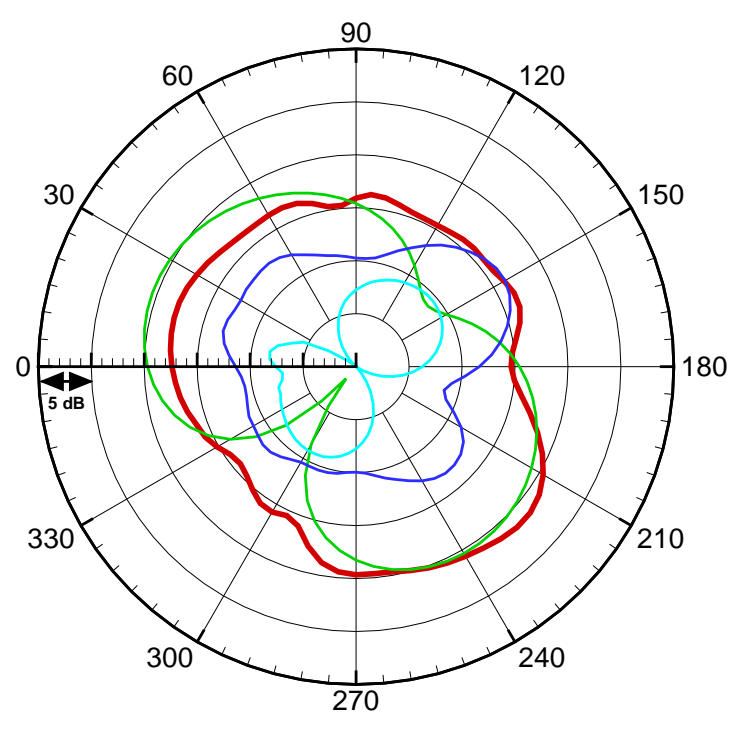

(a) all elements

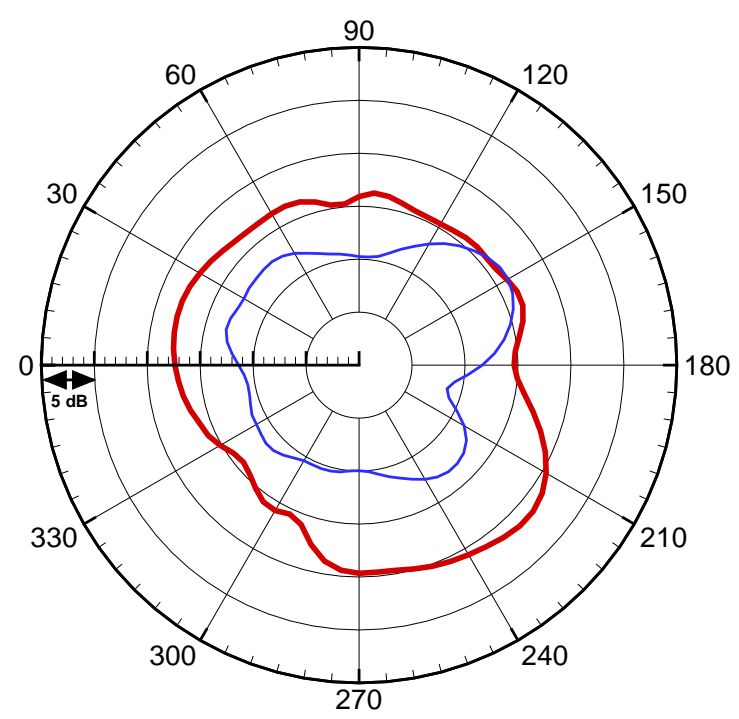

(c) wing

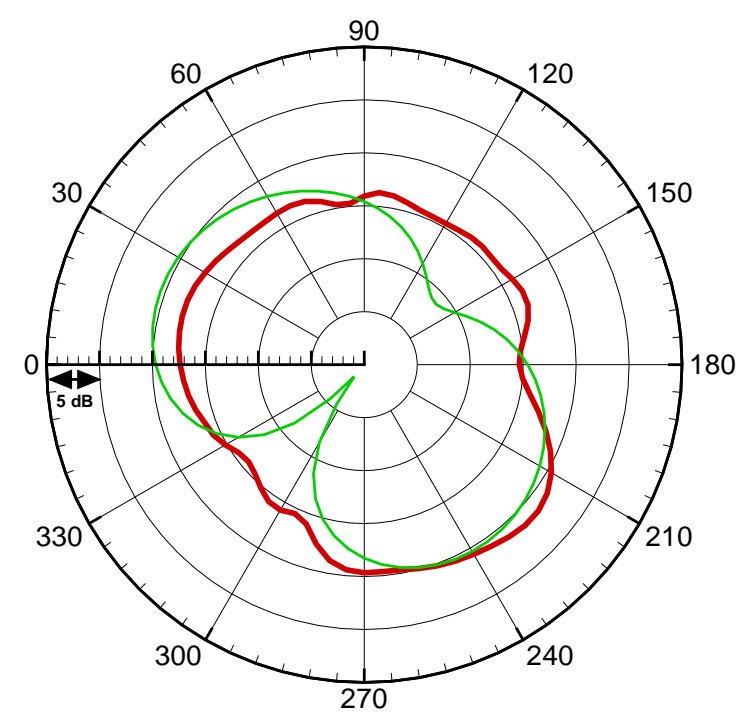

(b) slat

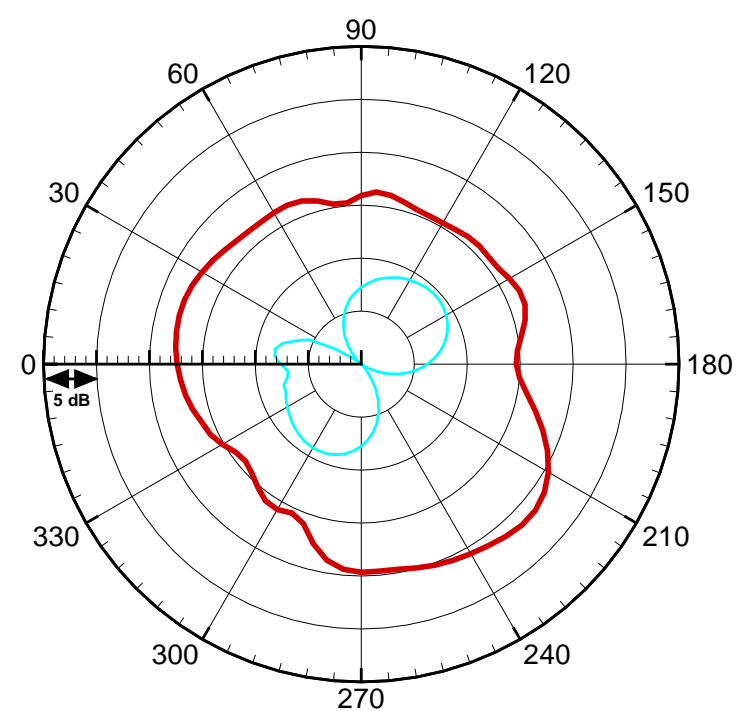

(d) flap

Figure 20: OASPL for the Curle method. $p_{\text {total }, C}^{\prime}$, $p_{\text {slat }, C}^{\prime}$ $p_{\text {wing }, C}^{\prime}$ $p_{\text {flap }, C}^{\prime}$

observe that at almost all frequencies the highest peaks are obtained with the Kirchhoff and FWH methods, which is consistent with the indications of the SPL and OASPL plots above.

In Figs. 15 and 17 a broad banded peak at large Strouhal numbers was presented, particularly in the upstream and downstream directions. Even though those two directions are of limited practical interest, the Strouhal number spectrum for the $\theta=0^{\circ}$ direction is given in Fig. 24. Even though the spectrum is dominated by the large peaks at low Strouhal numbers, some peaks can be identified at Strouhal numbers of about 35-45. As highlighted in the zoom-up of the high-frequency peaks presented in Fig. 24(b), the Kirchhoff method yields higher peaks and a larger contribution to the total noise than the FWH method. Moreover, one can observe that no peaks can be identified for the Curle analogy, as expected from Fig. 19. 


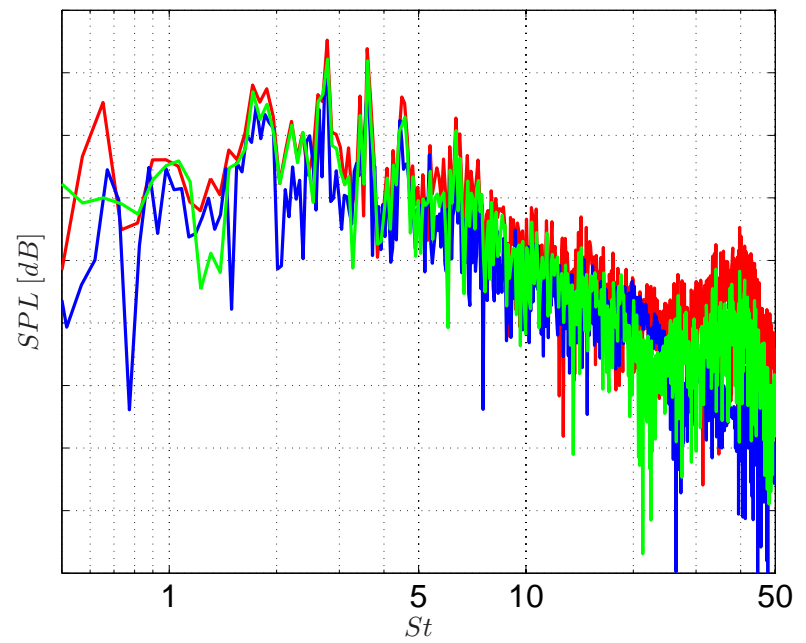

(a) $\theta=0^{\circ}$

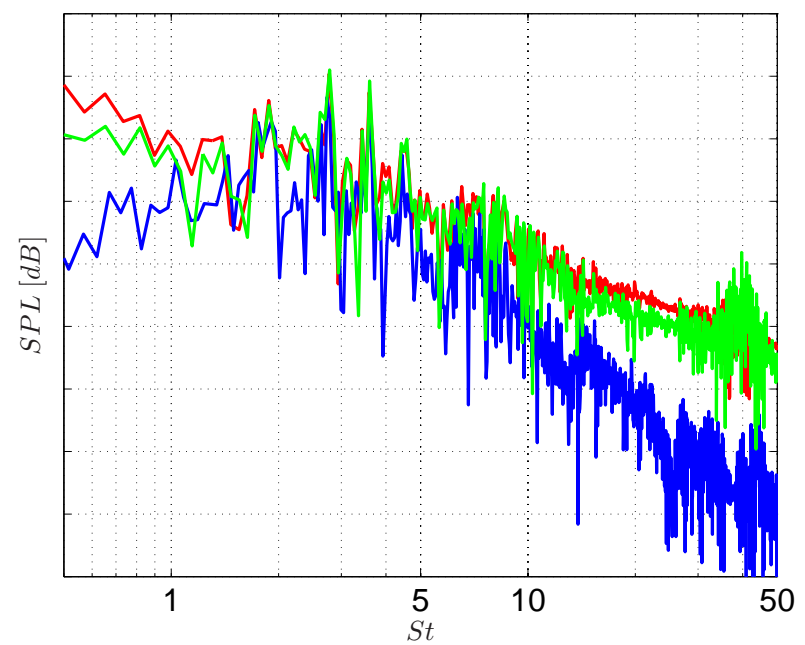

(c) $\theta=180^{\circ}$

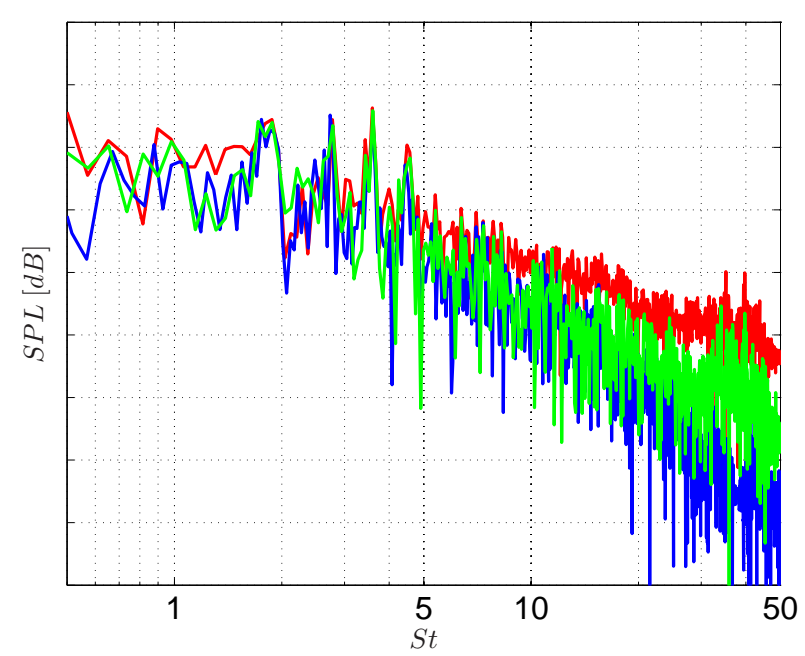

(b) $\theta=90^{\circ}$

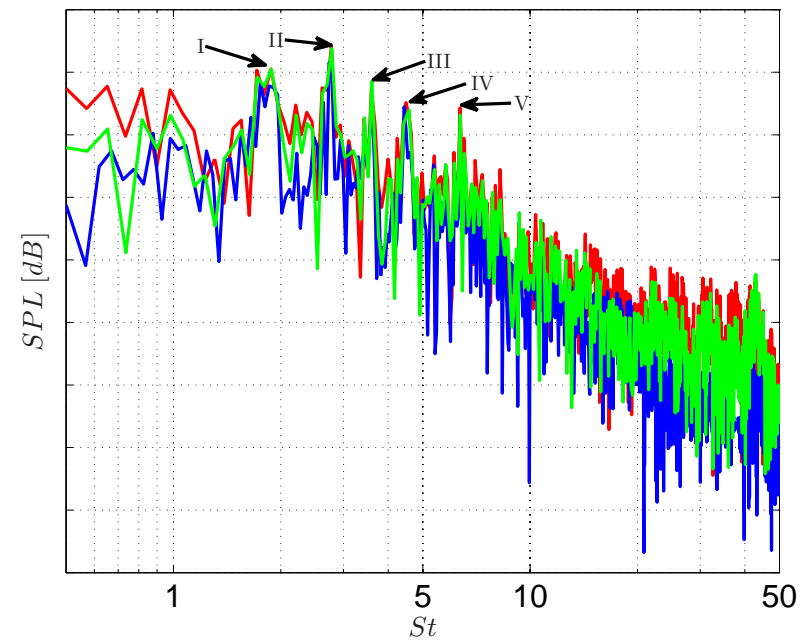

(d) $\theta=270^{\circ}$

Figure 21: Comparison of the SPL obtained with different acoustic analogies. Kirchhoff, Curle, : FWH. $\triangle S P L=10[d B]$. 


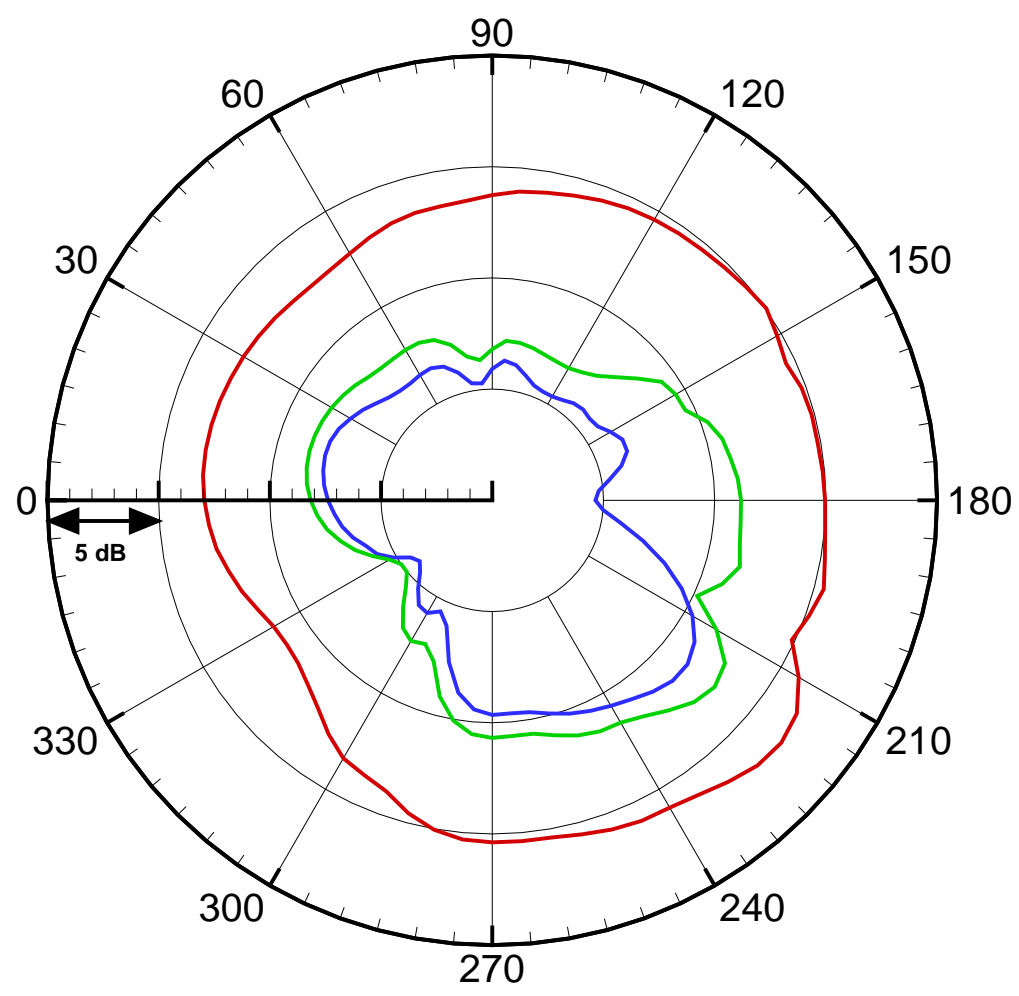

(a)

Figure 22: OASPL for the three acoustic analogies. Kirchhoff,

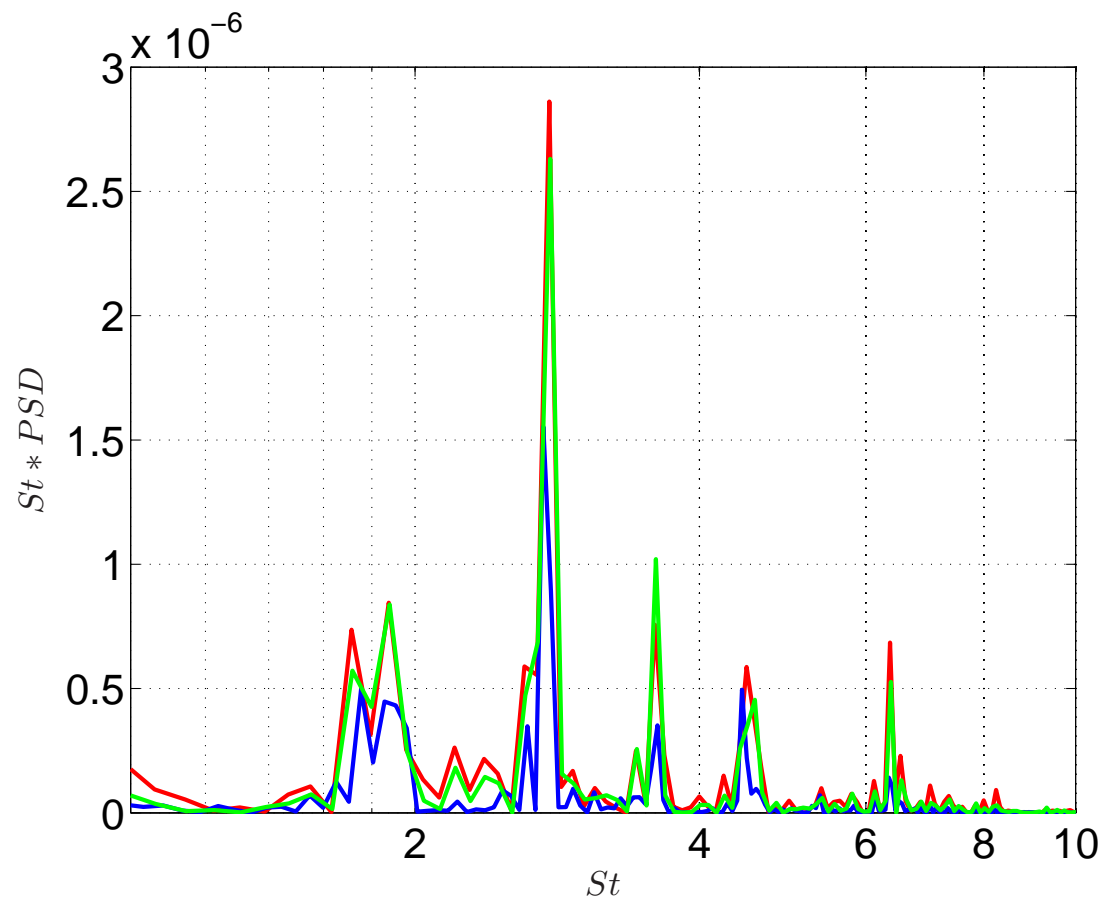

(a)

Figure 23: Energy spectrum of the sound pressure at $\theta=270^{\circ}$. Kirchhoff, Curle, FWH 


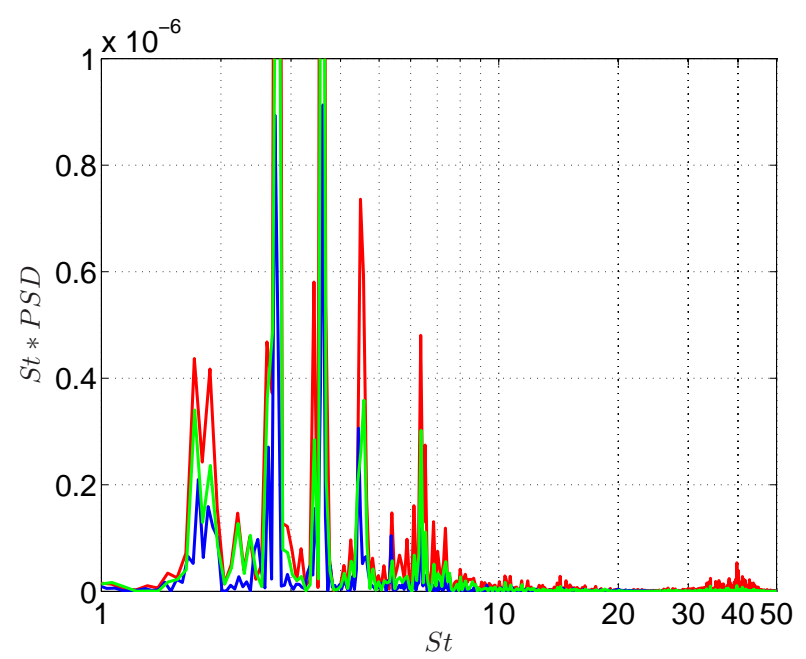

(a) entire spectrum

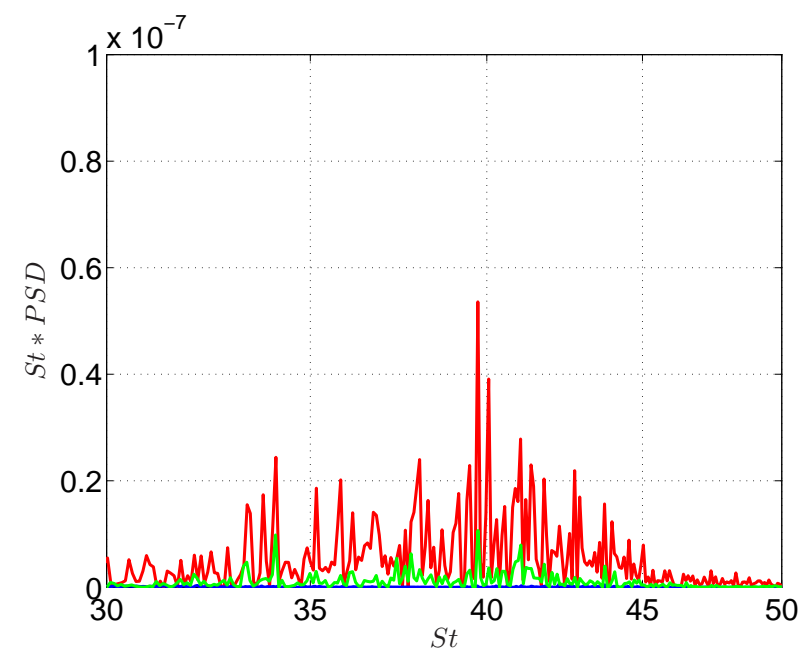

(b) zoom-in

Figure 24: Energy spectrum of the sound pressure at $\theta=0^{\circ}$.

Kirchhoff,

Curle,

: FWH 


\section{Conclusion}

The complex flow around a three-element high-lift airfoil configuration was investigated with the help of a hybrid RANS/LES modeling approach based on a simple algebraic RANS formulation. The pressure distribution around the three elements of the airfoil are in good agreement with experimental data. Due to the use of a finer grid in the $x-y$ plane, Kelvin-Helmholtz instabilities are observed almost immediately after the slat cusp, proving the superiority of the refined grid compared to the grid used in a previous study. However, secondary instabilities leading to fully three-dimensional turbulence in the shear layer are still delayed considerably. Due to that, the levels of resolved turbulent kinetic energy in the shear layer are still low in comparison with results shown by other research groups. Two point correlations in spanwise direction indicate that the spanwise domain extent of $16 \% C$ is sufficient. The used hybrid model has proved to be generally capable of predicting the flow around a high-lift airfoil. The current work is further evidence for the fact that with the help of hybrid RANS/LES modeling turbulence resolving methods are becoming more and more feasible for industrial applications.

An acoustic analysis of the far-field noise involving three acoustic analogies, namely the Kirchhoff, the Ffowcs-Williams and Hawkings and the Curle method, has been performed. For input data sampling for the source terms of the Kirchhoff and Ffowcs-Williams and Hawkings methods, a stationary, but permeable integral surface has been employed. All three methods agree well in terms of the frequencies at which tonal noise has been predicted. Using the Kirchhoff and FWH analogies, it was possible to capture the broad banded highfrequency peak, which is typically associated with vortex shedding from the blunt slat trailing edge. Once again, the slat could be established as the main contributor to airframe noise of high-lift airfoils. It was furthermore shown that both the slat and the flap radiate noise in a dipole fashion in the directions orthogonal to their own orientation.

\section{$5 \quad$ Future work}

The geometry should be computed with an improved hybrid RANS/LES model allowing for a faster development of the shear layer instabilities. Recent tests with a high-pass filtered SGS model in the LES region have shown that the levels of eddy viscosity can be efficiently reduced, which subsequently should lead to reduced dissipation and hence less hampered shear layer instabilities. Consequently also the propagation of acoustic waves should be less dampened.

A new experimental campaign should be started in an acoustical wind tunnel in order to provide accurate experimental noise levels, which can serve as an external reference for the assessment of the acoustic analogies. Furthermore, also the capabilities of the used hybrid RANS/LES modeling approach in terms of providing the required input data for the acoustic analogies could be evaluated.

\section{Acknowledgments}

The financial support of SNIC (the Swedish National Infrastructure for Computing) for computer time at C3SE (Chalmers Center for Computational Science and Engineering) is gratefully acknowledged. The authors also want to express their gratitude towards PDC (Center for High Performance Computing) at KTH Stockholm for allocated computer time. This project was financed by the EU project ATAAC (Advanced Turbulence Simulation for Aerodynamic Application Challenges), Grant Agreement No. 233710. The ATAAC project is described at http://cfd.mace.manchester.ac.uk/ATAAC/WebHome 


\section{References}

[1] INTERNATIONAL CIVIL AVIATION ORGANIZATION. Annex 16 to the Convention on International Civil Aviation, Volume 1 Aircraft Noise. ICAO, 4th edition, 2005.

[2] W. Dobrzynski, K. Nagakura, B. Gehlhar, and A. Buschbaum. Airframe Noise Studies on Wings with Deployed High-Lift Devices. In 4th AIAA/CEAS Aeroacoustics Conference, Toulouse, France, 2-4 June 1998. AIAA paper 98-2337.

[3] W. Dobrzynski and M. Pott-Pollenske. Slat Noise Source Studies for FarField Noise Prediction. In 7th AIAA/CEAS Aeroacoustics Conference, Maastricht, The Netherlands, 28-30 May 2001. AIAA paper 2001-2158.

[4] W. Dobrzynski, B. Gehlhar, and H. Buchholz. Model and full scale high-lift wing wind tunnel experiments dedicated to airframe noise reduction. Aerospace Science and Technology, 5:27-33, 2001.

[5] Y.P. Guo and M.C. Joshi. Noise Characteristics of Aircraft High-Lift Systems. AIAA Journal, 41:1247$1256,2003$.

[6] L.C. Chow, K. Mau, and H. Remy. Landing Gears and High Lift Devices Airframe Noise Research. In 8th AIAA/CEAS Aeroacoustics Conference and Exhibit, Breckenridge, USA, 17-19 June 2002. AIAA paper 2002-2408.

[7] B.A. Singer, D.P. Lockard, and K.S. Brentner. Computational Aeroacoustic Analysis of Slat Trailing-Edge Flow. AIAA Journal, 38:1558-1564, 2000.

[8] D.P. Lockard and M.M. Choudhari. Noise Radiation from a Leading-Edge Slat. In 15th AIAA Aeroacoustics Conference, Miami, USA, 11-13 May 2009. AIAA paper 2009-3101.

[9] H.-D. Yao, L.-E. Eriksson, L. Davidson, O. Grundestam, S.-H. Peng, and P.E. Eliasson. Aeroacoustic Assessment of Conceptual Low-Noise High-Lift Wing Configurations. In 50th AIAA Aerospace Sciences Meeting incl. the New Horizons Forum and Aerospace Exposition, Nashville, USA, 09-12 January 2012. AIAA paper 2012-0383.

[10] H.-D. Yao, L. Davidson, L.-E. Eriksson, O. Grundestam, S.-H. Peng, and P.E. Eliasson. Surface Integral Analogy Approaches to Computing Noise Generated by a 3D High-Lift Wing Configuration. In 50th AIAA Aerospace Sciences Meeting incl. the New Horizons Forum and Aerospace Exposition, Nashville, USA, 09-12 January 2012. AIAA paper 2012-0386.

[11] M.M. Choudhari and M.R. Khorrami. Effect of Three-Dimensional Shear-Layer Structures on Slat Cove Unsteadiness. AIAA Journal, 45:2174-2186, 2007.

[12] J. Wild, M. Pott-Pollenske, and B. Nagel. An integrated design approach for low noise exposing high-lift devices. In 3rd AIAA Flow Control Conference, San Francisco, USA, 2006. AIAA paper 2006-2843.

[13] J. Wild, G. Wichmann, F. Haucke, I. Peltzer, and P. Scholz. Large scale separation flow control experiments within the German Flow Control Network. In 47th AIAA Aerospace Sciences Meeting, Orlando, USA, 2009. AIAA paper 2009-0530.

[14] M.R. Khorrami, M.E. Berkman, and M. Choudhari. Unsteady Flow Computations of a Slat with a Blunt Trailing Edge. AIAA Journal, 38:2050-2058, 2000.

[15] L.N. Jenkins, M.R. Khorrami, and M.M. Choudhari. Characterization of Unsteady Flow Structures Near Leading-Edge Slat: Part I. PIV Measurements. In 10th AIAA Aeroacoustics Conference, Manchester, UK, 10-12 May 2004. AIAA paper 2004-2801.

[16] M.R. Khorrami, M.M. Choudhari, and L.N. Jenkins. Characterization of Unsteady Flow Structures Near Leading-Edge Slat: Part II. 2D Computations. In 10th AIAA Aeroacoustics Conference, Manchester, UK, 10-12 May 2004.

[17] S. Olson, F.O. Thomas, and R.C. Nelson. A Preliminary Investigation into Slat Noise Production Mechanisms in a High-Lift Configuration. In 18th AIAA Applied Aerodynamics Conference, Denver, USA, 14-17 August 2000. AIAA paper 2000-4508. 
[18] B.L. Storms, J.A. Hayes, J.C. Ross, and P.J. Moriarty. Aeroacoustic measurements of slat noise on a three-dimensional high-lift system. In 5th AIAA/CEAS Aeroacoustics Conference, Greater Seattle, USA, 10-12 May 1999. AIAA paper 99-1957.

[19] P.R. Spalart, W-H. Jou, M. Strelets, and S.R. Allmaras. Comments on the Feasibility of LES for Wings and on a Hybrid RANS/LES Approach. In Proceedings of the First AFOSR International Conference on DNS/LES, pages 137-147, Ruston, USA, 1997. Greyden Press.

[20] S. Deck. Zonal-Detached-Eddy Simulation of the Flow Around a High-Lift Configuration. AIAA Journal, 43:2372-2384, 2005.

[21] S. Deck. Recent improvements in the Zonal Detached Eddy Simulation (ZDES) formulation. Theor. Comput. Fluid Dyn., pages 1-28, 2011. 10.1007/s00162-011-0240-z.

[22] S. Deck. Numerical Simulation of Transonic Buffet over a Supercritical Airfoil. AIAA Journal, 43:15561566, 2005.

[23] F.R. Menter. Two-Equation Eddy-Viscosity Turbulence Models for Engineering Applications. AIAA Journal, 32:1598-1605, 1994.

[24] F.R. Menter, M. Kuntz, and R. Langtry. Ten Years of Industrial Experience with the SST Turbulence Model. In K. Hanjalic, Y. Nagano, and M. Tummers, editors, Turbulence, Heat and Mass Transfer 4, Antalya, Turkey, 2003. Begell House.

[25] L.G. Margolin and W.J. Rider. The design and construction of implicit LES models. International Journal for Numerical Methods in Fluids, 47(10-11):1173-1179, 2004. DOI: 10.1002/fld.862.

[26] L.G. Margolin, W.J. Rider, and F.F. Grinstein. Modeling turbulent flow with implicit LES. Journal of Turbulence, 7(15), 2006.

[27] A. Aspden, N. Nikiforakis, S. Dalziel, and J.B. Bell. Analysis of Implicit LES Methods. Communications in Applied Mathematics and Computational Science, 3(1):103-126, 2008.

[28] F. Farassat and M.K. Myers. Extension of Kirchhoff's Formula to Radiation from Moving Surfaces. Journal of Sound and Vibration, 123:451-460, 1988.

[29] J.E. Ffowcs-Williams and D.L. Hawkings. Sound Generation by Turbulence and Surfaces in Arbitrary Motion. Philosophical Transactions of the Royal Society, 264:321-342, 1969.

[30] K.S. Brentner and F. Farassat. Modeling aerodynamically generated sound of helicopter rotors. Progress in Aerospace Sciences, 39:83-120, 2003.

[31] N. Curle. The influence of solid boundaries upon aerodynamic sound. Proceedings of the Royal Society of London. Series A, Mathematical and Physical Sciences, 231:505-514, 1955.

[32] S.-H. Peng. Hybrid RANS-LES Modeling based on Zero- and One-Equation Models for Turbulent Flow Simulation. In Proceedings of 4 th International Symposium on Turbulence and Shear Flow Phenomena, volume 3, pages 1159-1164, Williamsburg, USA, 27-29 June 2005.

[33] S.-H. Peng. Algebraic Hybrid RANS-LES Modelling Applied to Incompressible and Compressible Turbulent Flows. In 36th Fluid Dynamics Conference and Exhibit, San Francisco, USA, 5-8 June 2006. AIAA paper 2006-3910.

[34] J. Smagorinsky. General Circulation Experiments with the Primitive Equations. Monthly Weather Review, 91:99-164, 1963.

[35] B. Nebenführ, S.-H. Peng, and L. Davidson. Precursor RANS computations for the F15 three-element high-lift configuration. Technical Report FOI Memo 2865, Chalmers/FOI, 2010.

[36] B. Nebenführ, S.-H. Peng, and L. Davidson. Hybrid RANS-LES Simulation of Turbulent High-Lift Flow in Relation to Noise Generation. In S. Fu et al., editor, Progress in Hybrid RANS-LES Modelling, NNFM, volume 117. Springer, 2012.

[37] G. Kirchhoff. Zur Theorie der Lichstrahlen. Annalen der Physik, 254:663-695, 1883. 
[38] A.S. Lyrintzis. Review: The Use of Kirchhoff's Method in Computational Aeroacoustics. Journal of Fluids Engineering, 116:665-676, 1994.

[39] M.J. Lighthill. On Sound Generated Aerodynamically. I. General Theory. Proceedings of the Royal Society of London. Series A, Mathematical and Physical Sciences, 211:564-587, 1952.

[40] M.J. Lighthill. On Sound Generated Aerodynamically. II. Turbulence As A Source Of Sound. Proceedings of the Royal Society of London. Series A, Mathematical and Physical Sciences, 222:1-32, 1954.

[41] K.S. Brentner and F. Farassat. An Analytical Comparison of the Acoustic Analogy and Kirchhoff Formulation for Moving Surfaces. AIAA Journal, 336:1379-1386, 1998.

[42] P.R. Spalart. Detached-Eddy Simulation. Annual Reviews of Fluid Mechanics, 41:181-202, 2009.

[43] F.R. Menter and M. Kuntz. Adaption of eddy-viscosity turbulence models to unsteady separated flow behind vehicles. In R. McCallen, F. Browand, and J. Ross, editors, Symposium on the aerodynamics of heavy vehicles: trucks, buses and trains., Monterey, USA, 2-6 December 2002. Springer.

[44] L. Davidson. Large Eddy Simulations: How to evaluate resolution. International Journal of Heat and Fluid Flow, 30:1016-1025, 2009.

[45] M.M. Choudhari, D.P. Lockard, M.G. Macaraeg, B.A. Singer, C.L. Streett, G.R. Neubert, R.W. Stoker, J.R. Underbrink, M.E. Berkman, M.R. Khorrami, and S.S. Sadowski. Aeroacoustic Experiments in the Langley Low-Turbulence Pressure Tunnel, 2002. NASA/TM-2002-211432.

[46] M.R. Khorrami. Understanding Slat Noise Sources. In Computational Aeroacoustics: From Acoustic Sources Modeling to Far-Field Radiated Noise Prediction Colloquium EUROMECH 449, Chamonix, France, 9-12 December 2003.

[47] C. Norberg. An experimental investigation of the flow around a circular cylinder: influence of aspect ratio. Journal of Fluid Mechanics, 258:287-316, 1994. doi:10.1017/S0022112094003332. 